\title{
Anomalous large-time behaviour of the $p$-Laplacian flow in an exterior domain in low dimension
}

\author{
Received February 1, 2008 and in revised form April 11, 2008
}

\begin{abstract}
We study the large time behaviour of weak nonnegative solutions of the $p$-Laplace equation posed in an exterior domain in space dimension $N<p$ with boundary conditions $u=0$. The description is done in terms of matched asymptotics: the outer asymptotic profile is a dipole-like self-similar solution with a singularity at $x=0$ and anomalous similarity exponents. The inner asymptotic behaviour is given by a separate-variable profile. We gather both estimates in a global approximant and we also study the behaviour of the free boundary for compactly supported solutions. We complete in this way the analysis made in a previous work for high space dimensions $N \geq p$, a range in which the large-time influence of the holes is less dramatic.
\end{abstract}

Keywords. $p$-Laplacian equation, exterior domain, asymptotic behaviour, domain with holes, matched asymptotics, anomalous self-similarity

\section{Introduction}

We are concerned with understanding the effect of the presence of one or several holes in the domain on the large-time behaviour of the solutions of nonlinear diffusion equations. In this paper we study the question for the evolution $p$-Laplace equation and find interesting non-standard asymptotics. To be specific, we consider an exterior domain $\Omega=\mathbb{R}^{N} \backslash \bar{G}$ where $G$ is a bounded domain in $\mathbb{R}^{N}$ with smooth boundary, and study the asymptotic behaviour of the solutions of the exterior Dirichlet problem with zero boundary conditions:

$$
\begin{cases}u_{t}=\Delta_{p} u, & (x, t) \in Q=\Omega \times(0, \infty), \\ u(x, t)=0, & (x, t) \in \partial \Omega \times(0, \infty), \\ u(x, 0)=u_{0}(x), & x \in \Omega .\end{cases}
$$

We also assume that $u_{0} \in L^{1}(\Omega), u_{0}$ is nonnegative, and $p>2$. By standard properties of the theory of the $p$-Laplacian equation the solution will be bounded for all $t>0$, hence we may also assume that $u_{0}$ is bounded in the study of large-time behaviour. The study

R. G. Iagar: Departamento de Matemáticas, Universidad Autónoma de Madrid, 28049 Madrid, Spain, and Institute of Mathematics of the Romanian Academy, P.O. Box 1-764, RO-014700, Bucureşti, Romania; e-mail: razvan.iagar@uam.es

J. L. Vázquez: Departamento de Matemáticas, Universidad Autónoma de Madrid, 28049 Madrid, Spain; e-mail: juanluis.vazquez@uam.es

Mathematics Subject Classification (2010): 35B40, 35K65, 35R35 
of the cases where $N \geq p$ was the object of a companion paper [14], where it was proved that for $N>p$ the large-time influence of the holes is moderate since the outflow through $\partial \Omega$ does not exhaust the whole initial mass $\int u_{0}(x) d x$ as $t \rightarrow \infty$, and the asymptotic profiles are still of the same type as in the Cauchy problem (Barenblatt profiles). For $N=p$ the mass goes to zero but the asymptotic rates and profiles can be considered as a limit case of the previous situation with the inclusion of logarithmic factors.

We devote this paper to the case of low dimension, $1 \leq N<p$, where the effect of the holes is more dramatic and the mathematical treatment more interesting: the asymptotic mass is zero and the renormalized asymptotic profiles correspond to what is known as self-similarity of the second kind, or self-similarity with anomalous exponents (see also [1]). Let us mention that such novel features do not appear in the study of large-time behaviour of the solutions of the porous medium equation recently done in [4] and [11], though a number of other properties are common. This gives relevance to the paper as a dynamical study of large asymptotic perturbations of self-similar regimes. The proof of uniqueness of the rescaled asymptotic profile needs an involved topological argument.

We may assume that $0 \in G$. We will work in most of the paper under the assumption that $u_{0}$ has compact support. This is done partly for simplicity, partly because compactly supported solutions have free boundaries whose behaviour is an interesting topic that we investigate; at the end, we show that this is not an essential restriction for the theory with more general initial data.

Preliminaries. We refer to DiBenedetto's book [8] and our paper [14] for convenient preliminaries on the $p$-Laplacian evolution equation and its weak formulation. Let us recall here only the concepts of solution that we use. We put $Q_{T}=\Omega \times(0, T)$.

Definition 1.1. A function $u \in C\left((0, T]: W_{0}^{1, p}(\Omega)\right) \cap L^{\infty}\left(Q_{T}\right)$ is a weak solution of problem 1.1 on $[0, T]$ if for any test function $\Phi \in C^{2,1}\left(\overline{Q_{T}}\right)$ with compact support in $\overline{Q_{T}}$ and $\Phi=0$ on $\partial \Omega \times(0, T]$, it satisfies the integral identity

$$
\begin{aligned}
\int_{\Omega} u(x, t) \Phi(x, t) d x= & \int_{0}^{t} \int_{\Omega}\left(u(x, s) \Phi_{s}(x, s)-|\nabla u|^{p-2} \nabla u(x, s) \cdot \nabla \Phi(x, s)\right) d x d s \\
& +\int_{\Omega} u_{0}(x) \Phi(x, 0) d x
\end{aligned}
$$

for any $t \in[0, T]$. We say that $u$ is a weak solution of $[1.1)$ on $Q$ if it is a weak solution in the sense above on $[0, T]$ for any $T>0$.

Definition 1.2. A function $u \in C\left((0, T]: W_{0}^{1, p}(\Omega)\right) \cap L^{\infty}\left(Q_{T}\right)$ is a local weak solution of problem 1.1 on $[0, T]$ if for any test function $\Phi \in C^{2,1}\left(Q_{T}\right)$ with compact support in $Q_{T}$, it satisfies the integral identity

$$
\int_{0}^{T} \int_{\Omega}\left(u(x, t) \Phi_{t}(x, t)-|\nabla u|^{p-2} \nabla u(x, t) \cdot \nabla \Phi(x, t)\right) d x d t=0 .
$$


We define a weak subsolution [supersolution] by replacing in Definition 1.1 the equality sign by $\leq$ (resp. $\geq$ ) and considering only nonnegative test functions $\Phi$. In a similar way, we define a local weak subsolution [supersolution] by replacing in Definition 1.2 the equality sign by $\geq$ (resp. $\leq$ ). We will also use the notation $r=|x|$.

Concerning the case $N>p$, we have already proved in [14] that the asymptotic profile is a Barenblatt profile, given by the expression

$$
B_{C}(x, t)=t^{-\alpha_{1}} F_{C}(\eta), \quad \eta=|x| t^{-\beta_{1}},
$$

where the profile has the form

$$
F_{C}(\eta)=\left(C-k \eta^{p /(p-1)}\right)_{+}^{(p-1) /(p-2)}
$$

with the well-known constants

$$
\alpha_{1}=\frac{N}{N(p-2)+p}, \quad \beta_{1}=\frac{1}{N(p-2)+p}, \quad k=\frac{p-2}{p} \beta_{1}^{1 /(p-1)} .
$$

The constant $C=C\left(u_{0}\right)>0$ is determined by the asymptotic study. The situation is different in the limit case $N=p$. It is proved in the same paper that the influence of the hole on the solutions of the $p$-Laplace operator is stronger, but it can still be expressed in terms of the same asymptotic profiles after a more dramatic time scaling. More specifically, the asymptotic profile is given by a precise logarithmic correction of the Barenblatt profile. This parallels the case $N=2$ of the porous medium equation with any $m>0$ which was studied in [11].

We end these preliminary results with a correspondence relation between the radially symmetric solutions of the $p$-Laplacian equation and of the porous medium equations, introduced in [13]:

Proposition 1.1. Suppose $0<n<2$. Then the radially symmetric solutions $u$ and $\bar{u}$ of the porous medium equation, resp. the p-Laplacian equation, are related through the following transformation:

$$
\bar{u}_{\bar{r}}(\bar{r}, t)=K r^{(2 n-2) /(m+1)} u(r, t), \quad K=\left(\frac{(m n-n+2)^{2}}{m(m+1)^{2}}\right)^{1 /(m-1)},
$$

where the correspondence of the parameters is

$$
p=m+1, \quad N=\frac{(n-2)(m+1)}{n-m n-2}
$$

and the independent variables are related by $\bar{r}=r^{(m n-n+2) /(m+1)}$.

Here $n$ is the dimension in the porous medium case and the variables with bar are for the $p$-Laplacian case.

The dipole solution. In the study of the asymptotic behaviour to be performed in this paper we will need another self-similar solution of the $p$-Laplacian evolution equation that was introduced in the paper [13] with the name of dipole solution. Precisely, it is 
obtained implicitly, through the exact relation between the profiles of the self-similar solutions of the porous medium and the $p$-Laplace equation. The name dipole comes from its properties similar to those of the well-known dipole solution of the heat equation and the porous medium equation: the mass $M(t)=\int u(x, t) d x$ as $t \rightarrow 0$ of the solution becomes infinite but at any positive moment it is finite.

Here, we have to face another difficulty: the profile of the dipole solution is not explicit, as it happens for the Barenblatt solution, and we will work using only its properties deduced from the analysis made in [13]. We will now state the properties we need. We will denote a particular dipole solution by $D$. Using the notations in [13], we write

$$
D(x, t)=t^{-\alpha_{2}} F\left(x t^{-\beta_{2}}\right),
$$

where the self-similarity exponents satisfy the relation

$$
(p-2) \alpha_{2}+p \beta_{2}=1, \quad \alpha_{2}, \beta_{2}>0,
$$

but we do not have explicit expressions for them, as in the porous medium case. Actually, such exponents are called anomalous, since they are not obtained from some conservation law but as the existence of a special orbit of an associated ODE system (cf. [1]). More precisely, in our case, since we are looking for self-similar solutions of the general form $t^{-\alpha} f(\eta), \eta=|x| t^{-\beta}$, the ODE satisfied by the profile $f$ is

$$
\eta^{1-N}\left(\eta^{N-1}\left|f^{\prime}\right|^{p-2} f^{\prime}\right)^{\prime}+\alpha f+\beta \eta f^{\prime}=0,
$$

which can be transformed into an autonomous dynamical system (see Section 4 of [13]).

We will also denote by $k_{2}=\alpha_{2} / \beta_{2}$ the associated "eigenvalue". From [13], we deduce that to this eigenvalue corresponds a whole orbit of solutions of dipole type, and, moreover, all their profiles are obtained from a particular representative $F$ through a simple rescaling:

$$
F_{\lambda}(\eta)=\lambda^{p} F\left(\lambda^{2-p} \eta\right), \quad \forall \lambda>0,
$$

hence we will denote the members of this orbit by $F_{\lambda}$ (the profile), and $D_{\lambda}$ (the solution corresponding to the profile $F_{\lambda}$ ). We remark that the scaling is monotone in $\lambda$, in the sense that if $\lambda_{1}<\lambda_{2}$, then both the support and the height of $D_{\lambda_{1}}$ are less than those of $D_{\lambda_{2}}$. When the index $\lambda$ is missing, we will understand $\lambda=1$. Using Proposition 1.1 and the behaviour near $x=0$ of the corresponding solutions of the porous medium equation (see [3], [12] or [13] where the calculations are given), we also find that $F_{\lambda}(0)=0$, but its derivative is singular at $\eta=0$. More precisely, near $\eta=0$ we have $F^{\prime}(\eta) \sim$ $\eta^{-(N-1) /(p-1)}$, hence

$$
F(\eta) \sim \eta^{(p-N) /(p-1)} \quad \text { as } \eta \sim 0,
$$

and we will denote by $C_{\lambda}$ the limit

$$
C_{\lambda}=\lim _{\eta \rightarrow 0} \frac{F_{\lambda}(\eta)}{\eta^{(p-N) /(p-1)}}
$$

Moreover, the dipole profile exists in the sense of weak solution in the whole space only in dimension $N<p$; for $N>p$ the profile develops a singularity at $\eta=0$, and for 


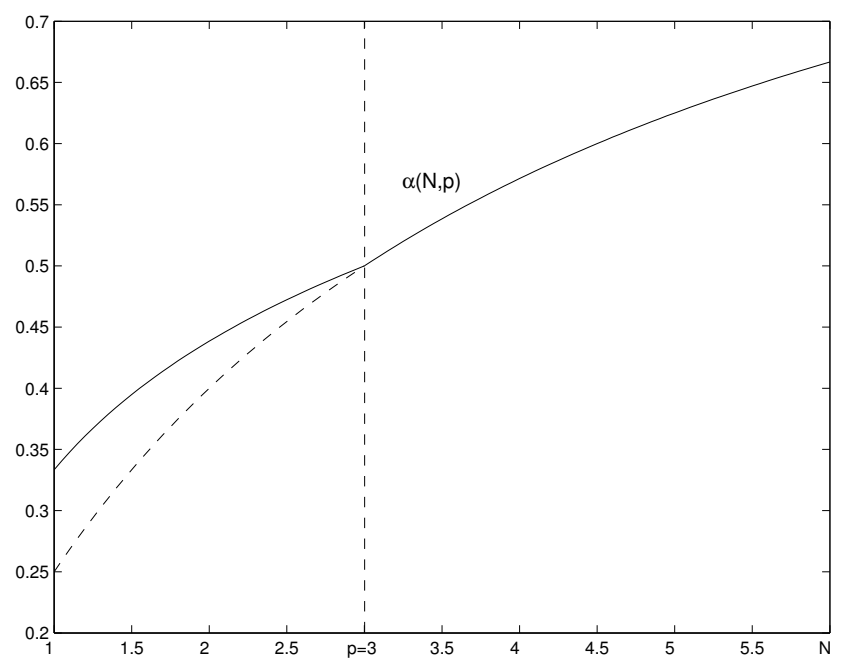

Fig. 1. Bifurcation of exponents at $N=p$. Experiment for $p=3$.

$N=p$ it coincides with the Barenblatt solution. We illustrate this bifurcation in Figure 1 below, where the dashed line represents the dipole exponents.

Even in dimension $N=1$ the dipole solution is not explicit. Actually, in that case, a simple differentiation leads to a self-similar solution of the porous medium equation with compactly supported profile and lap number 2. Bernis, Hulshof and Vázquez have studied that solution in [3] and shown that the similarity exponents are anomalous in the sense described above.

Outline of results. We begin by constructing in Section 2 the sub- and supersolutions that we will use later to obtain optimal barriers. Then we prove that the outer limit is given by a particular dipole profile by identifying the precise scaling factor $\lambda$ in $(1.12)$. The convergence to the outer profile is uniform in all sets of the form $\left\{|x| \geq \delta t^{\beta}\right\}$ for any $\delta>0$ sufficiently small. The main result is stated as Theorem 3.1 and the proof takes up Sections 3, 4 and 5. We will use a different technique than the one used in previous papers, like [4] or [14]. It is based on the construction of optimal barriers and delicate comparisons. An important step in the analysis is the proof of lack of contact between special solutions, which relies on a delicate use of the Harnack principle for degenerate parabolic equations with variable coefficients in space and time, due to [6]. The end steps rely on an accurate tail analysis. The whole process of proving uniqueness is much more difficult than for similar problems like in [4] where a conservation law is available to determine the asymptotic parameter. Here this law is replaced by a delicate topological study. The outer analysis ends with the convergence of the supports and interfaces, which follows as a consequence of the general uniqueness proof (Corollary 5.2).

The outer analysis is followed by the inner analysis in Section 6. The argument is similar to [14], based on an elliptic a priori bound and the technique of matched asymptotics. We show that the inner limit is given by a stationary state related to a particular solution 
of the following exterior Dirichlet problem:

$$
\begin{cases}\Delta_{p} H=0 & \text { in } \Omega \\ H=0 & \text { on } \partial \Omega .\end{cases}
$$

A global formulation and extensions to general $L^{1}$ data are treated in Section 7. We end the paper by proving new results for the porous medium equation as consequences of the present analysis and of the correspondence relations introduced in [13].

As a precedent of this work, a similar study was performed in a series of papers for another basic model of nonlinear diffusion, the porous medium equation, $u_{t}=\Delta u^{m}$, posed in an exterior domain in $\mathbb{R}^{n}$. It has been observed that the influence of the holes is very important in low dimensions. Thus, it was proved in [11] that in dimension $n<2$ there is a big difference in the asymptotic behaviour with respect to the case $n \geq 2$. In the latter, the asymptotic behaviour is given by the Barenblatt solution, but in the former the limit profile is the dipole solution. We point out that, since in the porous medium case the only subcritical dimension is $n=1$, the analysis in this case is similar to studying the porous medium equation on a half-line. On the contrary, in the case of the $p$-Laplacian evolution equation with $p$ large, there can be many space dimensions in the range $1 \leq$ $N<p$, making the analysis more interesting for applications.

\section{Sub- and supersolutions. Size estimates}

In this section we will construct appropriate sub- and supersolutions for our problem starting from the dipole profile that we have described before. Since from now on we will use only the dipole solutions, we will drop for simplicity the index 2 from the exponents $\alpha$ and $\beta$.

Supersolutions. We want to find a dipole solution $D_{\lambda}$ such that at $t=t_{0}>0$ fixed, $D_{\lambda}\left(x, t_{0}\right) \geq u\left(x, t_{0}\right)$. But using (1.12) and (1.13), we find that a general rescaled profile satisfies

$$
F_{\lambda}(\eta) \sim \lambda^{p-(p-2)(p-N) /(p-1)} \eta^{(p-N) /(p-1)} \rightarrow \infty \quad \text { as } \lambda \rightarrow \infty,
$$

and the convergence is uniform on compact sets far from the origin. On the other hand, the support of $F_{\lambda}$ tends to the whole space as $\lambda \rightarrow \infty$. Hence, if we fix $t_{0}>0$, there exists $\lambda>0$ sufficiently large such that

$$
D_{\lambda}\left(x, t_{0}\right) \geq u\left(x, t_{0}\right), \quad \operatorname{supp} u\left(\cdot, t_{0}\right) \subset \operatorname{supp} D_{\lambda}\left(\cdot, t_{0}\right) .
$$

By well-known comparison arguments, from 2.2 we deduce that the inequality holds at any later time, i.e. $u(x, t) \leq D_{\lambda}(x, t)$ for all $x \in \Omega$ and $t \geq t_{0}$.

Subsolutions. This case is much more difficult, since the dipole does not vanish on the boundary of $\Omega$. In order to construct a subsolution, we have to combine the dipole with 
another subsolution, using a technique similar to one in [14]. We define

$$
\begin{aligned}
D_{\lambda, \tau}(x, t) & =D_{\lambda}(x, t+\tau) \\
H_{\tau}(x, t) & =A(t)(t+\tau)^{-\alpha}\left(\left(\frac{|x|}{R_{0}}\right)^{(p-N) /(p-1)}-1-a \frac{\left(|x|-r_{1}\right)_{+}}{(t+\tau)^{l}}\right)_{+},
\end{aligned}
$$

where $\lambda, \tau, R_{0}, r_{1}, a, l$ are positive free parameters that have to be chosen. We choose $A(t)=2 K(t)$, where $K(t)=\max _{x} F_{\lambda}\left(x(t+\tau)^{-\beta}\right)$. It may be checked (by direct calculation) that $H_{\tau}$ is indeed a subsolution of the $p$-Laplacian equation in the whole $\mathbb{R}^{n}$.

Denote by $R_{1}(t)$ the radius of the interface of $H_{\tau}$, and $R_{2}(t)$ the radius of the interface of $D_{\lambda, \tau}$. We want $R_{2}(t)>R_{1}(t)$ for $t$ sufficiently large. We remark that $R_{2}(t) \sim(t+\tau)^{\beta}$ and $R_{1}(t)$ is a solution of the equation

$$
R_{1}(t)^{(p-N) /(p-1)}(t+\tau)^{l}=a R_{0}^{(p-N) /(p-1)}\left(R_{1}(t)-r_{1}\right)
$$

hence, after an easy calculation, $R_{1}(t) \sim(t+\tau)^{l(N-1) /(p-1)}$. Since $N<p$, it suffices to choose $l<\beta$ in order to get $R_{1}(t)<R_{2}(t)$ for $t \geq t_{0}$ sufficiently large. Hence, for any $t \geq t_{0}$, there exists $r^{*}(t)$ such that $1<r^{*}(t)<R_{1}(t)<R_{2}(t)$, so that the two subsolutions intersect at $|x|=r^{*}(t)$, with the correct angle of intersection (see Figure 2). Define

$$
V_{\lambda, \tau}(x, t)= \begin{cases}0 & \text { if } r<R_{0} \text { or } r>R_{2}(t) \\ H_{\tau}(x, t) & \text { if } R_{0} \leq|x| \leq r^{*}(t) \\ D_{\lambda, \tau}(x, t) & \text { if } r^{*}(t) \leq r \leq R_{2}(t)\end{cases}
$$

which is a well-defined subsolution of the problem (1.1) for $t \geq t_{0}$ sufficiently large. The following lemma shows that this family of subsolutions has good properties.

Lemma 2.1. There exists a choice of the parameters $\tau, \lambda, R_{0}, r_{1}, a, l$ and a time $t_{0}$ sufficiently large such that for $t \geq t_{0}$ we have

$$
V_{\lambda, \tau}(x, t) \leq u(x, t), \quad \forall x \in \Omega .
$$

Proof. We first show that there exists a time $t_{0}$ such that we have comparison at $t=t_{0}$. Fix $t_{0}$ large such that $V_{\lambda, \tau}$ is a subsolution. Choose first $l<\beta$, for example $l=\frac{1}{2} \beta$, and (by increasing $t_{0}$ if necessary) choose $R_{0}, r_{1}$ such that the annulus $\overline{W_{R_{0}, r_{1}}(0)}$ is included in the interior of $\operatorname{supp} u\left(\cdot, t_{0}\right)$. Then we choose $\lambda$, which measures the height of the subsolution $V_{\lambda, \tau}$, such that $V_{\lambda, \tau}$ lies below $u$ at time $t_{0}$. In order to choose the delay $\tau$, we require that

$$
\operatorname{supp} V_{\lambda, \tau}\left(\cdot, t_{0}\right)=\overline{W_{R_{0}, R_{2}(t)}(0)} \subset \operatorname{Int}\left(\operatorname{supp} u\left(\cdot, t_{0}\right)\right) .
$$

Hence we want to have $R_{2}\left(t_{0}\right)=\xi_{+}\left(t_{0}\right)-\varepsilon$, where

$$
\xi_{+}\left(t_{0}\right)=\sup \left\{r>0: B(0, r) \subset \operatorname{supp} u\left(\cdot, t_{0}\right)\right\} .
$$

But this implies a unique time $\tau$ for $t_{0}$ sufficiently large and $\varepsilon>0$ small. Finally, in order to choose $a$, we impose the condition $R_{1}\left(t_{0}\right)<R_{2}\left(t_{0}\right)$, and this implies a limitation on the value of $a$. 
We end the proof by showing that for any $t \geq t_{0}$, the inequality 2.5 holds. This follows from standard comparison arguments (the strong maximum principle) applied starting from $t=t_{0}$ as initial time. The only thing we need to check is that the previous construction can be done for $t>t_{0}$, and a necessary and sufficient condition is that $R_{1}(t)<R_{2}(t)$ for any $t>t_{0}$. But this holds true for sufficiently large $t_{0}$, due to the asymptotic rates of $R_{1}(t)$ and $R_{2}(t)$ and the fact that $l<\beta$.

We illustrate how the comparison is realized in Figure 2 below.

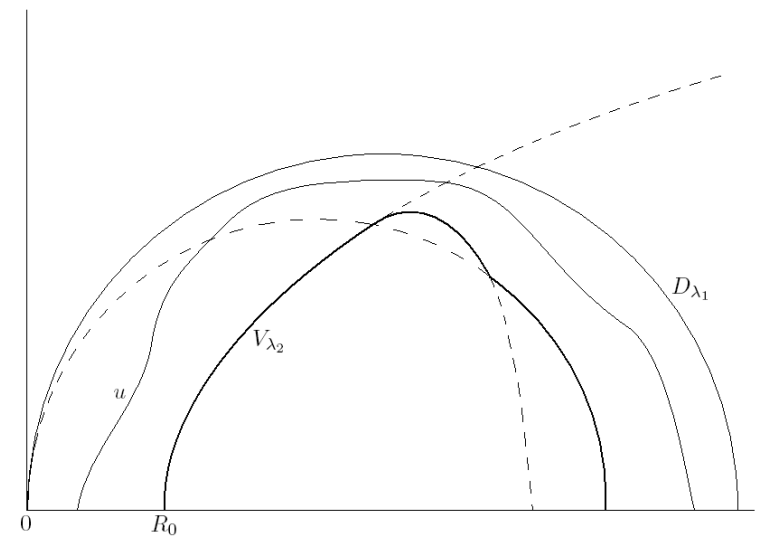

Fig. 2. Comparison of the solution $u$ with dipole profiles.

\section{Outer analysis I: Dipoles and the $\omega$-limit set}

In this section we introduce the concept of $\omega$-limit set of a renormalized orbit of a solution of problem (1.1) and relate it to a family of dipole solutions, which are our candidates for the asymptotic profile. The proof of the convergence to a particular dipole is long and delicate and will be continued in the next two sections. We fix the similarity exponents $\alpha$ and $\beta$ at the values introduced in $(1.10)$ for the dipole solutions. This is the main result:

Theorem 3.1. Let $1 \leq N<p$ and suppose also that $u_{0}$ is compactly supported. Then there exists a constant $\lambda>0$, depending on $N, p$ and the initial data $u_{0}$, such that

$$
\lim _{t \rightarrow \infty} t^{-\alpha}\left|u(x, t)-D_{\lambda}(x, t)\right|=0,
$$

with uniform convergence in all sets of the form $\left\{x \in \Omega:|x| \geq \delta t^{\beta}\right\}, \delta>0$.

The theorem will be proved using the technique of optimal barriers, also used in previous works like [10] for the porous medium equation or [17] for the Barenblatt equation of elasto-plastic filtration. The general idea of this technique is to construct the best barrier from above (or from below) for the asymptotic limit of the solution and then to show that in fact this optimal barrier equals the asymptotic limit, by using maximum and comparison principles. In the present case, the proof will be more involved than in these 
previous cases due to the degeneracy of the equation. This adds to the mathematical interest, specially in the analysis of contact points which can be seen as cases of application of non-standard strong maximum principles in the sense of [18]. Two final observations: (i) the same result 3.1 is true for general $L^{1}$ data, but this extension will be proved in Section 7; (ii) in the present case of compactly supported solutions, we can also prove convergence of the free boundaries: see Corollary 5.2 .

Let us proceed with the detailed proof. In the previous section, we have showed that there exist $\lambda_{1}$ and $\lambda_{2}$ such that

$$
V_{\lambda_{2}, \tau}(x, t) \leq u(x, t) \leq D_{\lambda_{1}}(x, t+\tau), \quad \lambda_{2}<\lambda_{1} .
$$

This allows us to define

$$
\lambda_{1}(t, \tau)=\inf \left\{\lambda_{1}: u(x, t) \leq D_{\lambda_{1}}(x, t+\tau), \forall x \in \Omega\right\} .
$$

The comparison argument for solutions implies that $\lambda_{1}$ is decreasing as a function of $t$. We may then define the asymptotic limit $\lambda_{1}(\infty, \tau)=\lim _{t \rightarrow \infty} \lambda_{1}(t, \tau)$ and $\lambda_{1}(\infty, \tau)>0$. In a similar way we may define

$$
\lambda_{2}(t, \tau)=\sup \left\{\lambda_{2}: V_{\lambda_{2}, \tau}(x, t) \leq u(x, t), \forall x \in \Omega\right\},
$$

hence the limit $\lambda_{2}(\infty, \tau)=\lim _{t \rightarrow \infty} \lambda_{2}(t, \tau)$ exists and it is easy to see that $\lambda_{2}(\infty, \tau) \leq$ $\lambda_{1}(\infty, \tau)$ for any $\tau>0$. The fact that the limit $\lambda_{1}(\infty, \tau)$ does not depend on the delay $\tau$ is a simple consequence of the following inequality satisfied by the dipole solutions.

Lemma 3.2. For any $\varepsilon>0$ small there exists $c(\varepsilon)>0$ such that whenever $\lambda>0$ and $|\tau|<c \varepsilon$,

$$
D_{\lambda}(x, t) \leq D_{\lambda(1+\varepsilon)}(x, t(1+\tau)), \quad \forall x \in \mathbb{R}^{N},
$$

Proof. By scaling $D_{\lambda}$ we may reduce the proof to the case $t=1$ and $\lambda=1$. Let us examine the case $\tau>0$. Away from $x=0$ this is geometrically easy. By increasing the parameter $\lambda=1$ to $1+\varepsilon$, the maximal height and the radius of the support increase. By inserting the delay in time, it is easy to see, from the definition of $D_{\lambda}$, that the maximal height decreases and the radius of the support increases.

It remains to show that the inequality holds near $x=0$. This follows from the estimate on the behaviour of the dipole at $x=0$ given in 1.13 and from the scaling formula (1.12). Indeed, in the first approximation near $x=0$, we have

$$
D_{1}(x, t) \sim C_{\lambda} t^{-\alpha-\beta(p-N) /(p-1)}|x|^{(p-N) /(p-1)},
$$

and

$D_{1+\varepsilon}(x, t+\tau) \sim C_{1+\varepsilon}(t+\tau)^{-\alpha-\beta(p-N) /(p-1)}(1+\varepsilon)^{p-(p-2)(p-N) /(p-1)}|x|^{(p-N) /(p-1)}$.

It is now easy to check that, fixing $t=1$, there exists $\tau_{1}=\tau_{1}(\varepsilon)$ such that the conclusion holds for any $0<\tau<\tau_{1}$, and the relation between $\tau_{1}$ and $\varepsilon$ is linear for $\varepsilon \approx 0$. A similar argument near $x=0$ will be used later to separate the contact at the origin.

The case $\tau<0$ is geometrically easier and we leave it to the reader. 


\subsection{Scalings, $\omega$-limit sets and optimal bounds}

For our next step we recall that the asymptotic analysis will depend on rescalings and limits. The rescaling that we will be using repeatedly is

$$
\left(T_{\gamma} u\right)(x, t)=\gamma^{\alpha} u\left(\gamma^{\beta} x, \gamma t\right)
$$

with exponents as in 1.10 ; we will often write $u_{\gamma}(x, t)$ instead of $\left(T_{\gamma} u\right)(x, t)$ for brevity. This rescaling keeps each of the dipole solutions $D_{\lambda}$ unchanged, and when applied to a solution $u$, the whole family $\left\{T_{\gamma} u=u_{\gamma}\right\}$ consists of solutions of the $p$-Laplacian equation. Moreover, the inequality in Section 2 becomes

$$
V_{\lambda_{2}, \tau / \gamma}(x, t) \leq\left(T_{\gamma} u\right)(x, t) \leq D_{\lambda_{1}}(x, t)
$$

for all $t \geq t_{0} / \gamma$. By the compactness estimates in [8], we can extract a subsequence $\left\{T_{\gamma_{k}} u\right\}$ converging to a limit $U_{\infty}$ as $\gamma_{k} \rightarrow \infty$; it is easy to see that this $U_{\infty}$ is a local weak solution of the $p$-Laplacian equation in $\left(\mathbb{R}^{N} \backslash\{0\}\right) \times(0, \infty)$, and the convergence is uniform on compact subsets of $\mathbb{R}^{N} \backslash\{0\}$. The limit function $U_{\infty}$ can (and will) have a singularity at $x=0$, and there could in principle be different limits depending on the chosen subsequence.

Following the dynamical systems terminology, we denote by $\omega(u)$ the $\omega$-limit set of the orbit $u(t)$, i.e., the set of all asymptotic limits of sequences $u_{\gamma_{k}}$ as $\gamma_{k} \rightarrow \infty$. A generic element of $\omega(u)$ will be denoted by $U$. Using 3.5 and the fact that through our rescaling, the delay disappears in the limit, we find that

$$
D_{\lambda_{2}(\infty, \tau)}(x, t) \leq U(x, t) \leq D_{\lambda_{1}(\infty, \tau)}(x, t)
$$

for all $U \in \omega(u), \tau>0$ and $x \in \mathbb{R}^{N} \backslash\{0\}$. Hence, we can reduce $\tau$ to 0 in the previous inequality. It now follows from standard theory that $\omega(u)$ is a bounded, closed and connected set in the space $C(Q)$ of continuous functions for every $Q=\mathbb{R}^{N} \times\left[t_{1}, t_{2}\right]$ with $0<t_{1}<t_{2}<\infty$.

With this in mind, we define the optimal dipole parameter from above as

$$
\lambda^{*}=\inf \left\{\lambda>0: U(x, t) \leq D_{\lambda}(x, t) \text { in } Q=\mathbb{R}^{N} \times(1 / 2, \infty) \text { for all } U \in \omega(u)\right\},
$$

where we look at $U$ and $D_{\lambda}$ as extended by zero at the origin. Obviously, $\lambda_{2}(\infty, \tau) \leq$ $\lambda^{*} \leq \lambda_{1}(\infty)$. In a similar manner, if we fix $U \in \omega(u)$, we can associate to it an upper optimal parameter $\lambda_{U}$ defined as

$$
\lambda_{U}=\inf \left\{\lambda>0: U(x, t) \leq D_{\lambda}(x, t) \text { in } Q=\mathbb{R}^{N} \times(1 / 2, \infty)\right\} .
$$

The pair $\left(U, D_{\lambda_{U}}\right)$ will be called an optimal pair. It is obvious that $\lambda_{U} \leq \lambda^{*}$ for any $U \in \omega(u)$; moreover, it is also easy to remark that

$$
\lambda^{*}=\sup \left\{\lambda_{U}: U \in \omega(u)\right\} .
$$

On the other hand, for any $U$ there exists a unique optimal pair $\left(U, D_{\lambda}\right)$, due to the fact that the family $\left\{D_{\lambda}\right\}$ is strictly increasing with respect to $\lambda$.

We will next prove a series of results in order to show that $D_{\lambda^{*}}$ is the unique element of $\omega(u)$, which will also end the proof of Theorem 3.1 Let us remark first that, from the definition of $\lambda^{*}$, we have $U \leq D_{\lambda^{*}}$ for any $U \in \omega(u)$. 


\section{Outer analysis II: Contact points and separation}

In this section we analyze in detail the optimal pairs $\left(U, D_{\lambda_{U}}\right)$ introduced in the previous section. As an intermediate step in our asymptotic analysis, we want to prove that $U=$ $D_{\lambda_{U}}$. Arguing by contradiction, if there is one $U \in \omega(u)$ that does not coincide with $D_{\lambda_{U}}$, then at least $U \leq D_{\lambda_{U}}$, and there could be three types of isolated contact points between $U$ and $D_{\lambda_{U}}$ :

(a) contact at a point $P=(x, t)$ which is not critical for $D_{\lambda_{U}}$;

(b) contact at the spatial maximum point (hot spot) of $D_{\lambda_{U}}$;

(c) contact on the free boundary of the two functions.

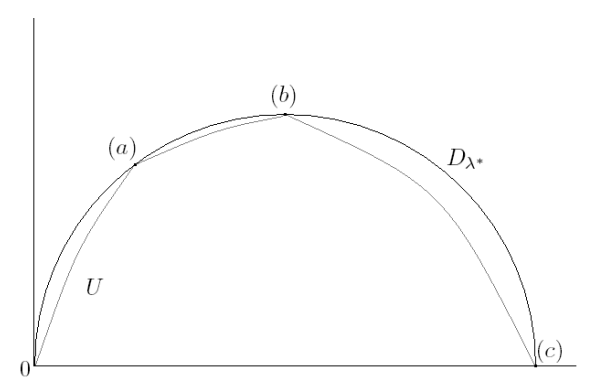

Fig. 3. Contact points of types (a), (b) and (c).

We will refer to these types of contact points as contact points of type (a), (b), (c) respectively (see the sketch in Figure 3). In what follows we prove that all the three types of contact points stated above either imply exact equality or are impossible (disappear) after finite time.

Lemma 4.1. A contact of type (a) implies equality.

Proof. Since the contact point is not a critical point for $D_{\lambda_{U}}$, this is an immediate consequence of the strong maximum principle for the $p$-Laplacian equation at nondegenerate points. We recall that weak solutions of the $p$-Laplacian evolution equation are $C^{1, \alpha}$ smooth with respect to the $x$ variable (see [8]).

\subsection{Analysis of a type (b) contact. The strong maximum principle}

In order to handle a contact point of type (b), where the equation degenerates for the solutions under consideration, we use the Harnack inequality proved by F. Chiarenza and R. Serapioni in [5] and improved in [6], for linear degenerate parabolic equations of the type

$$
u_{t}=\operatorname{div}(a(x, t) \nabla u) .
$$

We recall that the result holds if the matrix $a(x, t)$ may be degenerate but it is controlled in terms of a Muckenhoupt weight [7]. More precisely, it satisfies the following technical 
assumptions around some fixed point $\left(x_{0}, t_{0}\right)$ : there exists a non-negative function $\omega(x, t)$ defined on $\mathbb{R}^{N} \times(0, \infty)$ and some positive constant $\Gamma$ such that

$$
\omega(x, t)|\xi|^{2} \leq \sum_{i, j=1}^{N} a_{i, j}(x, t) \xi_{i} \xi_{j} \leq \Gamma \omega(x, t)|\xi|^{2}
$$

for a.e. $(x, t) \in Q$ and $\xi \in \mathbb{R}^{N}$, where $Q=\Omega \times(0, T)$, with $\Omega \subset \mathbb{R}^{N}$ open, and the function $\omega(x, t)$ is an $A_{2}$ weight in the time variable uniformly in $x$ and an $A_{1+2 / N}$ weight in $x$ uniformly in time, i.e., it satisfies the two conditions:

$$
\left(\frac{1}{|B|} \int_{B} \omega(x, t) d x\right)\left(\frac{1}{|B|} \int_{B} \omega(x, t)^{-N / 2} d x\right)^{2 / N} \leq c_{0}, \quad \forall t>0,
$$

and

$$
\left(\frac{1}{|I|} \int_{I} \omega(x, t) d t\right)\left(\frac{1}{|I|} \int_{I} \omega(x, t)^{-1} d t\right) \leq c_{0}, \quad \forall x,
$$

for some $c_{0}>0$, where $B$ represents any ball centred at $\left(x_{0}, t_{0}\right)$ with sufficiently small radius and $I \subset(0, \infty)$ any small time interval. Thus, the Harnack inequality holds on some special cylinders, depending on the degeneracy of the operator around the point. A precise definition of these cylinders is given in [6. Definition 3.2], and the Harnack inequality is proved as Theorem 3.4 of the same paper [6]. We apply this result to the analysis of our contact point.

Lemma 4.2. A contact of type (b) is impossible at any time $t>0$ unless there is equality for all $x$ and all later times.

Proof. (i) Linearization. Remember that we are assuming that $U$ and $D_{\lambda_{U}}$ are not identically equal. Suppose that we have a contact of type (b), so that $\nabla U=\nabla D_{\lambda_{U}}=0$ at $\left(x_{0}, t_{0}\right)$. Set

$$
w=U-D_{\lambda_{U}},
$$

which has an isolated zero at $\left(x_{0}, t_{0}\right)$ and it is a solution of the linearized equation

$$
w_{t}=\operatorname{div}(a(x, t) \nabla w),
$$

where

$$
a_{i j}(x, t)=\int_{0}^{1}|\nabla v(s)|^{p-4}\left((p-2) \partial_{i} v(s) \partial_{j} v(s)+|\nabla v(s)|^{2} I_{N}\right) d s
$$

is the matrix giving the degeneracy of the equation (4.6) in a parabolic neighbourhood $\mathcal{C}$ centred at $\left(x_{0}, t_{0}\right)$, where we denote

$$
v(s ; x, t)=\nabla D_{\lambda_{U}}+s\left(\nabla U-\nabla D_{\lambda_{U}}\right)
$$

and $I_{N}$ is the usual identity matrix. In what follows, we write $v(s)$ instead of $v(s ; x, t)$. Since the matrix $\left\{(p-2) \partial_{i} v(s) \partial_{j} v(s)\right\}_{i, j}$ is positive definite, it is sufficient to bound the 
second term from below, i.e. to have a bound from below for $\int_{0}^{1}|\nabla v(s)|^{p-2} d s$. On the other hand, the bound from above comes from the obvious inequality

$$
\sum_{i, j=1}^{N} a_{i j}(x, t) \xi_{i} \xi_{j} \leq(p-1) \int_{0}^{1}|\nabla v(s)|^{p-2} d s|\xi|^{2}, \quad \forall \xi \in \mathbb{R}^{N} .
$$

From these inequalities, we can take in (4.2)

$$
\omega(x, t)=\int_{0}^{1}|\nabla v(s)|^{p-2} d s \quad \text { and } \quad \Gamma=p-1
$$

for all $(x, t) \in \mathcal{C}$, extended in a nondegenerate way outside of the cylinder $\mathcal{C}$.

(ii) Lower estimate. We want to show that this weight satisfies in any case the conditions (4.3) and (4.4). First of all, we can bound it from below by terms depending only on estimates of $\left|\nabla D_{\lambda_{U}}\right|$, independent of the second term. At all points where $\nabla D_{\lambda_{U}} \neq 0$ we have

$$
\int_{0}^{1}\left|\nabla D_{\lambda_{U}}+s\left(\nabla U-\nabla D_{\lambda_{U}}\right)\right|^{p-2} d s=\left|\nabla D_{\lambda_{U}}\right|^{p-2} \int_{0}^{1}|a+s b|^{p-2} d s,
$$

where

$$
a=\frac{\nabla D_{\lambda_{U}}}{\left|\nabla D_{\lambda_{U}}\right|}, \quad b=\frac{\nabla U-\nabla D_{\lambda_{U}}}{\left|\nabla D_{\lambda_{U}}\right|},
$$

hence $a$ is a unit vector. By performing a rotation if necessary, we may assume that $a=$ $e_{1}$, the first vector of the canonical base of $\mathbb{R}^{N}$, hence we can work with scalar $a=1$ and $b$. But it is easy to see that if we define

$$
f(b)=\int_{0}^{1}|1+s b|^{p-2} d s,
$$

which admits a positive minimum as a function of $b$. We conclude that the matrix $\left\{a_{i j}(x, t)\right\}_{i, j}$ is bounded from below by $C\left|\nabla D_{\lambda_{U}}(x, t)\right|^{p-2}$ near $\left(x_{0}, t_{0}\right)$. Hence the worse possible degeneracy order at $\left(x_{0}, t_{0}\right)$ is that given by the dipole solution $\left|\nabla D_{\lambda_{U}}(x, t)\right|^{p-2}$. We deduce that, in order to check the conditions (4.3) and (4.4) on $\omega(x, t)$, it is sufficient if they hold for $\left|\nabla D_{\lambda_{U}}\right|^{p-2}$.

On the other hand, using the fact that $D_{\lambda_{U}}$ is a radial solution of the $p$-Laplacian equation and the correspondence relations between radial solutions of the $p$-Laplacian equation and the porous medium equation developed in [13], together with the behaviour of self-similar profiles of the porous medium equation near a point of change of sign given in [12], we obtain

$$
\left|\nabla D_{\lambda_{U}}(x, t)\right| \sim C\left|x-x_{0}\right|^{1 /(p-1)}
$$

near $\left(x_{0}, t_{0}\right)$, hence the maximal possible spatial degeneracy of (4.6) around $\left(x_{0}, t_{0}\right)$ is like $\left|x-x_{0}\right|^{(p-2) /(p-1)}$. 
(iii) $A_{p}$ conditions. We are now ready to check the conditions. The only problem is the behaviour of the last integrals near the line of degeneracy, $x(t)=x_{0}\left(t / t_{0}\right)^{\beta}$. It is easy to see that the maximal degeneracy with respect to the time variable near $\left(x_{0}, t_{0}\right)$ is like

$$
c\left|t^{\beta}-t_{0}^{\beta}\right|^{(p-2) /(p-1)} \sim c t_{0}^{\beta}\left|\left(t / t_{0}\right)^{\beta}-1\right|^{(p-2) /(p-1)} \sim c \beta t_{0}^{\beta}\left(t-t_{0}\right)^{(p-2) /(p-1)} .
$$

But a weight like $\Omega(x)=|x|^{(p-2) /(p-1)}$ satisfies condition 4.3 and a time weight like $c\left|t-t_{0}\right|^{(p-2) /(p-1)}$ satisfies 4.4$)$. Thus, the Harnack inequality ([6, Theorem 3.4]) applies and shows that $\inf _{\mathcal{C}} w>0$, where $\mathcal{C}$ is a small special cylinder around $\left(x_{0}, t_{0}\right)$, of type $\left\{\left|x-x_{0}\right|<r\right\} \times\left\{t_{0}-k\left(x_{0}, t_{0}, r\right)<t<t_{0}\right\}$ (see [6] for details), in particular there is no contact of type (b) between $U$ and $D_{\lambda_{U}}$ at times before $t_{0}$. Hence, we cannot have a contact of type (b) at any time (except in the trivial case when $U \equiv D_{\lambda_{U}}$, which we are assuming not to hold). Consequently, there exists a time $t_{0}>0$ such that we have no contact of type (b) at $\left(x_{0}, t_{0}\right)$.

(iv) Barrier argument. We end the proof by showing that there is no contact of type (b) at times after $t_{0}$. Since we have no such contact at $\left(x_{0}, t_{0}\right)$, there exists an annulus $r_{1}^{0}<|x|<r_{2}^{0}$, containing the maximum points of $D_{\lambda_{U}}$ at $t_{0}$ (i.e. with $|x|=\left|x_{0}\right|$ ), such that in this annulus we have a uniformly strict inequality $U\left(x, t_{0}\right)<D_{\lambda_{U}}\left(x, t_{0}\right)$. Consider $t \in\left[t_{0}, T\right]$, with $T<\infty$ arbitrary, and denote by $r(t)=r_{0}\left(t / t_{0}\right)^{\beta}$ the absolute value of the spatial maximum points of $D_{\lambda_{U}}(\cdot, t)$. Let $0<r_{1}(t)<r(t)<r_{2}(t)$ be such that $r_{1}\left(t_{0}\right)=r_{1}^{0}, r_{2}\left(t_{0}\right)=r_{2}^{0}$ and $r_{i}(t)$ is continuous for $t_{0} \leq t \leq T$. Since there is no contact of type (a) for $|x|=r_{1}(t)$ or $|x|=r_{2}(t)$, we have $U(x, t)<D_{\lambda_{U}}(x, t)$ uniformly. Since the map $\varepsilon \mapsto D_{\lambda_{U}-\varepsilon}$ is uniformly continuous, we find $\varepsilon>0$ (depending on $T$ ) sufficiently small such that

$$
D_{\lambda_{U-\varepsilon}}(x, t)>U(x, t)
$$

for $|x|=r_{i}(t), i=1,2, t_{0}<t \leq T$, and for $t=t_{0}, r_{1}^{0}<|x|<r_{2}^{0}$, i.e., in the whole parabolic boundary of a domain in $\mathbb{R}^{N+1}$. Hence, this inequality extends to the interior at any time $t \in\left(t_{0}, T\right)$. In other words, $U \leq D_{\lambda_{U}-\varepsilon}$ in the region $t_{0} \leq t \leq T$, $r_{1}(t)<|x|<r_{2}(t)$, and consequently $U$ lies strictly below $D_{\lambda_{U}}$. In particular, since $T$ was arbitrarily large, this shows that a contact of type (b) is impossible after $t_{0}$. Note finally that we can take $t_{0}$ as small as we please.

\subsection{Separation alternative}

We continue here the effort to prove that every $U \in \omega(u)$ is in fact a dipole solution. The proof will depend on whether the strong maximum principle at points of type (b) is uniform in the following sense.

Lemma 4.3. For any optimal pair $\left(U, D_{\lambda_{U}}\right)$ with $U \in \omega(u)$, the following alternative holds: either we have asymptotic separation

$$
\inf _{t>1,|x|=\left|x_{0}(t)\right|} t^{\alpha}\left(D_{\lambda_{U}}\left(x, t+\tau_{0}\right)-U(x, t)\right)>0,
$$

or $D_{\lambda_{U}} \in \omega(U)$. Moreover, in the latter case $D_{\lambda_{U}} \in \omega(u)$. 
Proof. Suppose that the infimum in the statement is 0 . Then there exists a sequence $\left\{t_{n}\right\}$ of times such that

$$
\lim _{n \rightarrow \infty} t_{n}^{\alpha}\left(D_{\lambda_{U}}\left(x, t_{n}+\tau_{0}\right)-U\left(x, t_{n}\right)\right)=0 .
$$

Using the rescaling 3.4 with $\gamma=t_{n}^{-1}$, we find that there exists a sequence $U_{n}=U_{t_{n}^{-1}}$ of rescaled versions of $U$ that converges to a limit $U^{*}$ which touches $D_{\lambda_{U}}$ at time $t=1$ and $|x|=\left|x_{0}(1)\right|$ (the existence of the limit follows from classical compactness estimates, see [8]). But from Lemma 4.2 this is not possible, unless $U^{*} \equiv D_{\lambda_{U}}$. This proves the statement. The fact that $D_{\lambda_{U}} \in \omega(u)$ follows easily by a standard diagonal argument.

Assume now that the strong separation 4.7 does not hold. In this case, we prove:

Lemma 4.4. If $D_{\lambda_{U}} \in \omega(U)$, then $U \equiv D_{\lambda_{U}}$. Consequently, any optimal pair reduces in this case to the dipole solution contained in it.

Proof. Let $\left\{u_{\gamma_{k}}\right\}$ be a subsequence converging to $U$. We prove first that the family $u_{\gamma_{k}}=$ $T_{\gamma_{k}} u$ becomes arbitrarily close to $D_{\lambda_{U}}$ for $k$ large, at time $t=1$ :

Claim. For any $\varepsilon>0$, there exists $k=k(\varepsilon)$ sufficiently large such that $u_{\gamma_{k}}=T_{\gamma_{k}} u>$ $D_{\lambda_{U}}-\varepsilon$ in $\Omega\left(\gamma_{k}\right):=\gamma_{k}^{-\beta} \Omega$, at time $t=1$, for all $k \geq k(\varepsilon)$.

Proof of the claim. Fix $t=1$ and suppose that the claim is false, hence there exists $\varepsilon_{0}>0$, a sequence $\left(k_{n}\right)$ going to infinity and $x_{n} \in \Omega$ such that $u_{\gamma_{k_{n}}}\left(x_{n}, 1\right)<$ $D_{\lambda_{U}}\left(x_{n}, 1\right)-\varepsilon_{0}$. Using the standard compactness estimates and passing to the limit, we find that there exists a compact set $K \subset \mathbb{R}^{N} \backslash\{0\}$ such that $U(x, 1)<D_{\lambda_{U}}(x, 1)-\varepsilon_{0}$ for all $x \in K$. On the other hand, by hypothesis, there exists a subsequence of rescaled versions of $U$ converging to $D_{\lambda_{U}}$. Then, by uniform continuity of the map $T_{\gamma}$, there exists a first $\gamma_{0}$ such that $U_{\gamma_{0}}(x, 1)+\varepsilon_{0} \geq D_{\lambda_{U}}(x, 1)$ in $\mathbb{R}^{N}$ and the two functions will touch. But their contact points are necessarily interior points for $D_{\lambda_{U}}$, since they are near the origin and near the free boundary $D_{\lambda_{U}}-\varepsilon_{0}<0$, and this is impossible from Lemmas 4.1 and 4.2 and the fact that $U_{\gamma_{0}} \in \omega(u)$.

Last argument. We choose $k$ sufficiently large such that $D_{\lambda_{U}}(x, 1) \leq \varepsilon$ for all $x \in$ $\partial \Omega\left(\gamma_{k}\right)$ and $k \geq k(\varepsilon)$. From 1.13 , we deduce that $D_{\lambda_{U}}(x, t) \leq \varepsilon$ for all $x \in \mathbb{R}^{N} \backslash \Omega\left(\gamma_{k}\right)$ and all $t>1$. We then compare $u_{\gamma_{k}}$ and $\tilde{u}=D_{\lambda_{U}}-\varepsilon$ in $Q_{k}=\Omega\left(\gamma_{k}\right) \times[1, \infty)$, where $k$ is large as in the previous step. Both are solutions of the original $p$-Laplacian equation (1.1). Moreover, the claim proved above gives us comparison at $t=1$ for any $k \geq k(\varepsilon)$, and the discussion above shows that $\tilde{u} \leq 0=u_{\gamma_{k}}$ on $\partial \Omega\left(\gamma_{k}\right)$ for all $t$. It follows from the maximum principle applied to the original equation that $u_{\gamma_{k}}(x, t) \geq \tilde{u}(x, t)=D_{\lambda_{U}}(x, t)-\varepsilon$ in $Q_{k}$ for all $k \geq k(\varepsilon)$. Passing to the limit in $k$, we obtain $U(x, t) \geq D_{\lambda_{U}}(x, t)-\varepsilon$ in $Q_{*}=\left(\mathbb{R}^{N} \backslash\{0\}\right) \times[1, \infty)$ for all $U \in \omega(u)$. Since $U \leq D_{\lambda_{U}}$ and $\varepsilon$ is arbitrarily small, we find that $U \equiv D_{\lambda_{U}}$, as desired.

As an immediate consequence, we find that if the strong separation assumption (4.7) does not hold, all the elements of $\omega(u)$ are necessarily dipole solutions with varying parameters. In the next subsection we essentially treat the complementary case, where the strong separation holds. 


\subsection{The case of strong separation}

We now study what happens if the separation assumption (4.7) holds.

Lemma 4.5. Let $\left(U, D_{\lambda_{U}}\right)$ be an optimal pair such that the strong separation assumption 4.7 holds. Then there exists $\widetilde{U} \in \omega(u)$ with $\lambda_{\tilde{U}}<\lambda_{U}$.

Proof. We start with the easier case where also the free boundaries of $U$ and $D_{\lambda_{U}}$ are separated. Then we show that the strong separation implies that we arrive at this situation in any case. We thus divide the proof into three steps.

Step 1. Assume for instance that the free boundaries of $U$ and $D_{\lambda_{U}}$ are separated at $t=t_{0}>0$. By rescaling we may assume that $t_{0}=1$. Using the separation Lemma 4.2 . we can take $\varepsilon>0$ sufficiently small such that $U(x, 1) \leq D_{\lambda_{U}-\varepsilon}(x, 1)$ for $|x| \geq x_{0}(t)$. We look for a small time advancement $\tau_{1}(\varepsilon)>0$ such that $U(x, 1) \leq D_{\lambda_{U}-\varepsilon / 2}\left(x, 1-\tau_{1}\right)$ for all $x$ near the origin. In order to find such a $\tau_{1}$, we recall the scaling $(1.12)$ and the behaviour of the dipole profiles near the origin given by 1.13 . It is enough to have $D_{\lambda_{U}-\varepsilon / 2}\left(x, 1-\tau_{1}\right) \geq D_{\lambda_{U}}(x, 1)$ for all $x \in \mathbb{R}^{N}$ with $|x|$ sufficiently small. Comparing their principal terms, we need

$$
\begin{aligned}
C_{\lambda_{U}-\varepsilon}\left(1-\tau_{1}\right)^{-\alpha-\beta(p-N) /(p-1)}\left(\lambda_{U}-\varepsilon / 2\right)^{p-(p-2)(p-N) /(p-1)}|x|^{(p-N) /(p-1)} \\
\\
\geq C_{\lambda_{U}}\left(\lambda_{U}\right)^{p-(p-2)(p-N) /(p-1)}|x|^{(p-N) /(p-1)},
\end{aligned}
$$

or equivalently,

$$
\left(1-\tau_{1}\right)^{\alpha+\beta(p-N) /(p-1)} \leq \frac{C_{\lambda_{U}-\varepsilon / 2}}{C_{\lambda_{U}}}\left(\frac{\lambda_{U}-\varepsilon / 2}{\lambda_{U}}\right)^{p-(p-2)(p-N) /(p-1)},
$$

which is the condition that $\tau_{1}$ should satisfy. By possibly decreasing $\varepsilon$, we find $\tau_{1}(\varepsilon)>0$ sufficiently small such that the above condition is satisfied and the free boundaries of $U(x, 1)$ and $D_{\lambda_{U}-\varepsilon / 2}\left(x, 1-\tau_{1}\right)$ are still separated. Then we easily get $U(x, 1) \leq$ $D_{\lambda_{U}-\varepsilon / 2}\left(x, 1-\tau_{1}\right)$ for all $x \in \mathbb{R}^{N}$. By standard comparison, we then find

$$
U(x, t) \leq D_{\lambda_{U}-\varepsilon / 2}\left(x, t-\tau_{1}\right) \quad \text { for } x \in \mathbb{R}^{N}, t>1 .
$$

By rescaling, we obtain

$$
U_{\gamma}(x, t) \leq D_{\lambda_{U}-\varepsilon / 2}\left(x, t-\tau_{1} / \gamma\right) \quad \text { for } x \in \mathbb{R}^{N}, t>1,
$$

for any $\gamma>0$. Passing to the limit in $\gamma$, we find in this case that $\omega(U)$ admits $D_{\lambda_{U}-\varepsilon / 2}$ as upper bound. Since $\omega(U) \subseteq \omega(u)$, there exists an element $\widetilde{U} \in \omega(u)$ (in fact any element of $\omega(U)$ is good in this sense) such that $\lambda_{\tilde{U}}<\lambda_{U}$.

Step 2. We will now assume that we are in the situation of a free boundary contact and that the strong separation assumption 4.7 holds. In that case we consider comparison of $U(x, t)$ and $D_{\lambda_{U}-\varepsilon}\left(x, t+\tau_{0} / 2\right)$ for some $\varepsilon>0$ in the region $Q_{+}=\{(x, t): t \geq 1,|x| \geq$ $\left.x_{0}(t)\right\}$. We choose $\varepsilon>0$ sufficiently small such that $D_{\lambda_{U}-\varepsilon}\left(x, 1+\tau_{0} / 2\right) \geq U(x, 1)$, 
ensuring in this way the comparison at our initial time $t=1$. The comparison on the lateral boundary $|x|=x_{0}(t)$ follows from the strong separation 4.7$)$. We conclude that

$$
D_{\lambda_{U}-\varepsilon}\left(x, t+\tau_{0} / 2\right) \geq U(x, t) \quad \text { in } Q_{+},
$$

hence their free boundaries are ordered,

$$
r[U](t) \leq r\left[D_{\lambda_{U}-\varepsilon}\right](t+\tau)=c\left(\lambda_{U}-\varepsilon\right)^{\sigma}(t+\tau)^{\beta}
$$

and for large times we get separation of the free boundaries of $U$ and $D_{\lambda_{U}}$, which leads us to the previous step.

Step 3. We conclude that the free boundary contact disappears if the separation assumption (4.7) holds, hence we can separate the free boundaries of $U$ and $D_{\lambda_{U}}$ for large times. After this, we arrive at the case in Step 1, hence the lemma is proved.

We remark that in this case we cannot conclude that $U \equiv D_{\lambda_{U}}$ directly, but the result of Lemma 4.5 will be used in the next section together with new arguments to arrive at that conclusion.

\section{Outer analysis III: Tail analysis and uniqueness}

In this section, we prove that $\omega(u)=\left\{D_{\lambda^{*}}\right\}$, finalizing in this way the proof of Theorem 3.1. From the previous analysis, we know that $\omega(u)$ contains only dipole solutions or solutions bounded from above by such dipoles (as follows from the strong separation alternative treated in the previous section). The main difficulty of proving that this set reduces in fact to a unique solution (for example the maximal one) is that the functions $u_{\gamma}$ could have a long thin tail, i.e. a region where $\left|u_{\gamma}\right| \leq \varepsilon$ is very small, but that region could be a priori very large. The existence of such a tail makes any comparison argument difficult, since the supports of the rescaled functions may be much greater than the supports of their limits. Hence, the analysis we do is based on elimination or reduction of such a tail.

\subsection{Bounds for the tail}

In a first step we show that the tail is not larger in the limit than the support of the maximal dipole $D_{\lambda^{*}}$. Denote by $r_{\gamma}(t, \theta)=r\left[u_{\gamma}\right](t, \theta)$ the maximum free boundary radius of $u_{\gamma}$ for fixed parameter $\gamma$, time $t$ and angle $\theta \in \mathbb{S}^{N-1}$. Likewise, we let $R_{\lambda}(t)=r\left[D_{\lambda}\right](t)=$ $c(\lambda, p, d) t^{\beta}$ be the maximum radius for $D_{\lambda}$, which does not depend on $\theta$. We denote $C(t, \theta):=\limsup _{\gamma \rightarrow \infty} r_{\gamma}(t, \theta)$. With these notations, we prove:

Lemma 5.1. For any $t>0$ and $\theta \in \mathbb{S}^{N-1}$, we have $C(t, \theta) \leq R_{\lambda^{*}}(t)=c_{*} t^{\beta}$.

Proof. (i) A preliminary consequence of scaling. We first prove that for any $t>0$ and $\theta \in \mathbb{S}^{N-1}$ fixed, we have $C(t, \theta)=C(1, \theta) t^{\beta}$. We can write $u_{\gamma}(x, t)=t^{-\alpha} u_{\gamma t}\left(x t^{-\beta}, 1\right)$. 
Since the same family appears in both members, passing to maximal radii we obtain

$$
r_{\gamma}(t, \theta)=r_{\gamma t}(1, \theta) t^{\beta}
$$

hence, by taking limits, we find $C(t, \theta)=t^{\beta} C(1, \theta)$. Below we write $C(1, \theta)=C$ for brevity.

(ii) Argument by contradiction. Suppose that the statement is false and there exist $t_{0}>0$ and $\theta_{0} \in \mathbb{S}^{N-1}$ such that $C\left(t_{0}, \theta_{0}\right)>R_{\lambda^{*}}\left(t_{0}\right)$. By the rescaling (i) we may assume that $t_{0}=1 / 2$. For simplicity, we take the direction $\theta_{0}$ of maximum $C\left(1 / 2, \theta_{0}\right)$ that we consider fixed from now on and write $C(t)=C\left(t, \theta_{0}\right)$ and $r_{\gamma}(t)=r_{\gamma}\left(t, \theta_{0}\right)$. The plan of the argument is to show that at time $t=1$, we have $C(1) \leq R_{\lambda^{*}}(1)$, which would contradict the original assumption in view of the power-like formulas $C(t, \theta)=C(1, \theta) t^{\beta}$ and $R_{\lambda^{*}}(t)=c_{*} t^{\beta}$.

Arguing by contradiction, suppose that $C(1)>R_{\lambda^{*}}(1)$. Passing to a subsequence, it follows that, for any $\varepsilon>0$, there exists $k=k(\varepsilon)$ and a subsequence $u_{\gamma_{k}}$ such that $r_{\gamma_{k}}(1) \geq C(1)-\varepsilon>R_{\lambda^{*}}(1)$ for all $k \geq k(\varepsilon)$.

Some notations: From part (i), it is immediate that $C(1 / 2)=C(1) / 2^{\beta}<C(1)$. By decreasing $\varepsilon>0$ if necessary, we may assume that $C(1)-3 \varepsilon>C(1 / 2)$ and at the same time $C(1)-3 \varepsilon>R_{\lambda^{*}}(1)$. Set $C_{0}:=\max \left\{C(1 / 2), R_{\lambda^{*}}(1)\right\}<C(1)-3 \varepsilon$. We are now ready for the main calculation.

(iii) Comparison with a travelling wave. Since the extra part of the supports of $u_{\gamma}$ takes the form of a thin tail, coming back to the subsequence $\gamma_{k}$ chosen above, we may assume that for $|x| \geq R_{\lambda^{*}}(t)+\varepsilon, t \in[1 / 2,1]$ and $k \geq k(\varepsilon)$ large, we have $\left|u_{\gamma_{k}}(x, t)\right| \leq \varepsilon$. In order to control the length of this tail region, we consider the travelling wave

$$
\hat{u}(x, t)=\left(\frac{p-2}{p-1}\right)^{(p-1) /(p-2)}\left(\varepsilon(t-1 / 2)+\varepsilon+C_{0}-x_{1}\right)_{+}^{(p-1) /(p-2)}
$$

and we compare it with $u_{\gamma_{k}}$ in the region $\left\{x_{1} \geq C_{0}, t \in[1 / 2,1]\right\}$ for $k \geq k(\varepsilon)$. Following the lines of the proof of Theorem 18.8 in [20] and rotating the argument, we find that $r_{\gamma_{k}}(1) \leq C_{0}+2 \varepsilon<C(1)-\varepsilon$, contradiction. Hence the supposition made in part (ii) is false and $C(1) \leq R_{\lambda^{*}}(1)$.

As an immediate consequence, if $\left\{u_{\gamma_{l}}\right\}$ is a subsequence converging to $D_{\lambda^{*}}$ (if such a subsequence exists), we obtain $r_{\gamma_{l}}(t, \theta) \rightarrow R_{\lambda^{*}}(t)$, since the estimate from below follows immediately from the locally uniform convergence. We need a convergence result for the free boundary under more general circumstances.

Lemma 5.2. Let $\left\{u_{\gamma_{k}}\right\}$ be a subsequence converging to $U \in \omega(u)$ with $\left(U, D_{\lambda_{U}}\right)$ an optimal pair, $\lambda_{U}<\lambda^{*}$. Then, for all $t>0$ and $\theta \in \mathbb{S}^{N-1}$,

$$
\lim _{k \rightarrow \infty} r_{\gamma_{k}}(t, \theta)=R_{\lambda *}(t)
$$


Proof. (i) Suppose that convergence from below is false and there exist $t_{0}, \gamma_{0}>0$ and some small $\varepsilon>0$ and $\tau_{0} \geq 0$ such that

$$
u_{\gamma_{0}}\left(x, t_{0}\right) \leq D_{\lambda^{*}-\varepsilon}\left(x, t_{0}-\tau_{0}\right), \quad x \in \Omega\left(\gamma_{0}\right) .
$$

By parabolic comparison between the solutions $u_{\gamma_{0}}$ and $D_{\lambda_{*}-\varepsilon}$, we find that $u_{\gamma_{0}}(x, t) \leq$ $D_{\lambda^{*}-\varepsilon}\left(x, t-\tau_{0}\right)$ for any $t>t_{0}$, and by inverting the scaling, we have $u(x, t) \leq$ $D_{\lambda^{*}-\varepsilon}\left(x, t-\tau_{0} \gamma_{0}\right)$ for any $t>t_{0} \gamma_{0}$. It follows that

$$
u_{\gamma}(x, t) \leq D_{\lambda^{*}-\varepsilon}\left(x, t-\tau_{0} \gamma_{0} / \gamma\right)
$$

for any $\gamma>0$ and $t>t_{0} \gamma_{0} / \gamma$. This contradicts the definition of $\lambda^{*}$, since any limit $U \in \omega(u)$ is bounded above by $D_{\lambda^{*}-\varepsilon}$.

(ii) From the uniform convergence to $U$, for any $\delta>0$, there exists $k=k(\delta)$ large such that $u_{\gamma_{k}}<D_{\lambda_{U}}+\delta$ on $\operatorname{supp}\left(D_{\lambda_{U}}\right) \cap \Omega\left(\gamma_{k}\right)$ for any $k \geq k(\delta)$. Hence, if

$$
\liminf _{k \rightarrow \infty} r_{\gamma_{k}}(t, \theta)<R_{\lambda^{*}}(t)
$$

then the situation in the previous paragraph can be realized for some $k$ very large (corresponding to $\delta$ small enough). Hence, the limit above should be at least $R_{\lambda^{*}}(t)$. Using also Lemma 5.1. we conclude that the limit is precisely $R_{\lambda^{*}}(t)$, for any $t>0$.

\subsection{Uniqueness of the limit profile. Final argument}

We can now show that $D_{\lambda^{*}}$ is the unique asymptotic limit. This will be a consequence of the following

Lemma 5.3. Let $\left(U, D_{\lambda_{U}}\right)$ be an optimal pair. Then necessarily $\lambda_{U}=\lambda^{*}$.

Proof. Suppose not and consider an optimal pair $\left(U, D_{\lambda_{U}}\right)$ with $\lambda_{U}<\lambda^{*}$. Then there exists a subsequence $u_{\gamma_{k}}$ converging to $U$. Now, we take up the technique of Lemma 5.1 and we want to compare the solutions $u_{\gamma_{k}}$ with a similar travelling wave as in 5.1 . In this case, we consider $t=1$ as starting time, $t=2$ as final time and define $C_{0}:=$ $\max \left\{R_{\lambda^{*}}(1), R_{\lambda_{U}}(2)\right\}$. Choose $\varepsilon>0$ so small that $C_{0}<R_{\lambda^{*}}(2)-3 \varepsilon$. The thin tail exists now at least for $|x| \geq R_{\lambda_{U}}(t)+\varepsilon, k$ large, $t \in[1,2]$, and in this region we may assume that $\left|u_{\gamma_{k}}(x, t)\right| \leq \varepsilon$. Then we define $\hat{u}$ as in 5.1), with our new $C_{0}$ and $\varepsilon$, and we compare $u_{\gamma_{k}}$ and $\hat{u}$ for $k \geq k(\varepsilon)$ sufficiently large. By a similar comparison as in the proof of Lemma 5.1. we find that $r_{\gamma_{k}}(2, \theta) \leq C_{0}+2 \varepsilon<R_{\lambda^{*}}(2)-\varepsilon$ for any $k \geq k(\varepsilon)$ sufficiently large and $\theta \in \mathbb{S}^{N-1}$. In conclusion, if there exists a subsequence converging to a limit $U$ bounded above by a dipole with parameter $\lambda_{U}<\lambda^{*}$, we are able, after a time, to decrease the tail (uniformly in $\theta$ ) with respect to the free boundary of $D_{\lambda^{*}}$. But this contradicts the result of Lemma 5.2

Corollary 5.1. The strong separation alternative obtained in Section 4 is impossible. 
Proof. If we have strong separation between $U$ and $D_{\lambda_{U}}=D_{\lambda^{*}}$, then from Lemma 4.5. there exists an optimal pair $\left(\widetilde{U}, D_{\lambda_{\tilde{U}}}\right)$ with parameter $\lambda_{\tilde{U}} \leq \lambda^{*}-\varepsilon / 2$, contrary to Lemma 5.3 .

It follows that necessarily $\omega(u)=\left\{D_{\lambda^{*}}\right\}$ and Theorem 3.1 is finally proved. We also deduce, as an immediate consequence, that

$$
\lim _{\gamma \rightarrow \infty} r_{\gamma}(t, \theta)=R_{\lambda^{*}}(t)
$$

for any $t>0$, uniformly in $\theta \in \mathbb{S}^{N-1}$. This implies the convergence of supports and interfaces of the general solution $u$ to those of $D_{\lambda^{*}}$. Indeed, if we introduce the notations

$$
r_{+}(t)=\max _{x \in \Gamma(t)}|x|, \quad r_{-}(t)=\min _{x \in \Gamma(t)}|x|,
$$

where $\Gamma(t)$ is the free boundary of the solution $u$ at time $t$, then from 5.2 and the usual rescaling, we can state the following:

Corollary 5.2. In the conditions of Theorem 3.1 and with notations of the previous paragraphs, we have

$$
\lim _{t \rightarrow \infty} \frac{r_{ \pm}(t)}{R_{\lambda^{*}}(t)}=1
$$

Remark. Since $F(\eta) \sim \eta^{(p-N) /(p-1)}$, we see that the dipole solution is a local weak solution of the $p$-Laplacian evolution equation in $\left(\mathbb{R}^{N} \backslash\{0\}\right) \times(0, \infty)$ with $N<p$, in the sense specified in Definition 1.2. but it is not a weak solution in the sense of Definition 1.1 Indeed, from the flux condition

$$
\lim _{\eta \rightarrow 0} \eta^{N-1}\left|F^{\prime}(\eta)\right|^{p-1}=0
$$

we see that if $F(\eta) \sim \eta^{\gamma}$ as $\eta \rightarrow 0$, then the self-similar solution whose profile is $F(\eta)$ is a weak solution for $\gamma>(p-N) /(p-1)$. This shows that the singularity at $x=0$ of the limit function cannot be removed for $N<p$ (as it happens for $N>p$, see [14]).

\section{Inner analysis}

The ideas are similar to those in the first part of this study (see [14]). In order to apply the technique of matched asymptotics, we start by multiplying the solution with the correct scale in time. Since we want the matching to take place on the curve $|x|=\delta t^{-\beta}$, and the expected result is a stationary solution, we derive that the correct scale of time is $t^{\alpha+(p-N) \beta /(p-1)}$. We set

$$
\bar{w}(x, t)=t^{\alpha+\frac{p-N}{p-1} \beta} u(x, t) .
$$

Then $\bar{w}$ satisfies the equation:

$$
\Delta_{p} \bar{w}=t^{-\frac{(N+1) p-2 N}{p-1} \beta}\left(t \bar{w}_{t}-\left(\alpha+\frac{p-N}{p-1} \beta\right) \bar{w}\right) .
$$


Suppose for the moment that the term on the right-hand side of 6.2 tends to 0 as $t \rightarrow \infty$. Then the asymptotic limit of $\bar{w}$ is expected to be a solution of the stationary Dirichlet problem

$$
\begin{cases}\Delta_{p} v=0 & \text { in } \Omega \\ v=0 & \text { on } \partial \Omega .\end{cases}
$$

As is well-known, all the solutions of this elliptic problem tend to infinity with a rate $|x|^{(p-N) /(p-1)}$ as $|x| \rightarrow \infty$ and they have the general form $C H_{p}$, where $H_{p}$ is the unique solution of the problem 6.3 having the additional property

$$
\lim _{|x| \rightarrow \infty} \frac{H_{p}(x)}{|x|^{(p-N) /(p-1)}}=1 .
$$

In fact, if $\Omega$ is the exterior of a ball of radius $R$, this solution is $H_{p}(x)=|x|^{(p-N) /(p-1)}-$ $R^{(p-N) /(p-1)}$. In the general case, it is easy to prove the behaviour of $H_{p}$ stated above, by comparing it with radial sub- and supersolutions.

We use the method of matched asymptotics (see [14]) in order to find the precise constant $C$. From the outer analysis result, we have

$$
\lim _{t \rightarrow \infty} t^{\alpha}\left|u(x, t)-D_{\lambda_{0}}(x, t)\right|=0,
$$

uniformly for all $x \in \Omega$ with $|x| \geq \delta t^{\beta}$, where $\lambda_{0}$ is the scaling parameter in 1.12 corresponding to the limit of $u$. On the other hand, from our formal deduction we expect that

$$
\lim _{t \rightarrow \infty}\left|t^{\alpha} u(x, t)-\frac{C H_{p}(x)}{t^{\beta(p-N) /(p-1)}}\right|=0
$$

uniformly for $x \in \Omega$ with $|x| \leq \delta t^{\beta}, \delta>0$ small. By comparing the two limits on the curve $|x|=\delta t^{\beta}$, we obtain

$$
\frac{C H_{p}\left(\delta t^{\beta}\right)}{t^{\beta(p-N) /(p-1)}} \sim t^{\alpha} D_{\lambda_{0}}\left(\delta t^{\beta}, t\right)=F_{\lambda_{0}}(\delta) \quad \text { as } t \rightarrow \infty .
$$

Since the last term of this equivalence is independent of time, by choosing $\delta$ sufficiently small we have $F_{\lambda_{0}}(\delta) \sim C_{\lambda_{0}} \delta^{(p-N) /(p-1)}$ (recall the notation $C_{\lambda}$ from 1.14)). By passing to the limit formally as $\delta \rightarrow 0$ and taking into account the behaviour of $H_{p}$, we find that necessarily $C=C_{\lambda_{0}}$.

In what follows, we will prove rigorously that the inner asymptotic behaviour of the solution $u$ of 1.1 is given by the separate variable function $C_{\lambda_{0}} H_{p}(x) / t^{\beta(p-N) /(p-1)}$. We use the technique introduced in [14] and based on an optimal elliptic a priori bound proved also in [14], which we recall in its more general form:

Proposition 6.1. Let $\Omega \subset \mathbb{R}^{N}$ be a bounded domain, $f \in C(\Omega) \cap L^{\infty}(\Omega), g \in C(\partial \Omega)$ and $u \in C^{1}(\Omega) \cap C(\bar{\Omega})$ be the solution of the Dirichlet problem

$$
\begin{cases}\Delta_{p} u=f & \text { in } \Omega \\ u=g & \text { on } \partial \Omega .\end{cases}
$$


Then there exists a constant $C>0$, independent of the diameter of $\Omega$, such that

$$
|u| \leq C d^{p /(p-1)}\left(\sup _{\Omega}|f|\right)^{1 /(p-1)}+\sup _{\partial \Omega}|g| \quad \text { in } \Omega,
$$

where $d=\operatorname{diam}(\Omega)$.

We change the scale of time by setting $\tau=\log t$ and we define

$$
w(x, t)=\frac{\bar{w}(x, t)}{t^{\beta(p-N) /(p-1)}}=t^{\alpha} u(x, t)=e^{\alpha \tau} u\left(x, e^{\tau}\right) .
$$

Consider the time averages

$$
W_{T}(x, \tau)=\frac{1}{T} \int_{\tau}^{\tau+T} w(x, s) d s .
$$

We first show that the time averages converge, that is:

Proposition 6.2. For any $\varepsilon>0$ and $T>0$, there exists a constant $\delta=\delta(\varepsilon, T)>0$ and a large time $\tau_{\mathrm{in}}=\tau_{\mathrm{in}}(\varepsilon, \delta, T)$ such that for any $\tau \geq \tau_{\text {in }}$ we have

$$
\left|W_{T}(x, \tau)-\frac{C_{\lambda_{0}} H_{p}(x)}{t^{\beta(p-N) /(p-1)}}\right| \leq \varepsilon
$$

for all $x$ with $|x| \leq \delta e^{\beta \tau}$.

Proof. The proof follows closely those of Propositions 2.3 and 3.4 in [14]. From the outer analysis result, we deduce that for any $\varepsilon, \delta>0$, there exists a time $\tau_{0}=\tau_{0}(\varepsilon, \delta)>0$ sufficiently large such that

$$
F_{\lambda_{0}}(\delta)-\varepsilon \leq w(x, t) \leq F_{\lambda_{0}}+\varepsilon
$$

for all $\tau \geq \tau_{0}$ and $|x|=\delta t^{\beta}$. Denote, as in [14], $R(\tau)=\delta e^{\tau \beta}$ and $\Omega_{\tau}=\Omega \cap B(0, R(\tau))$ and set $g=\left.w\right|_{\partial B(0, R(\tau))}$. Then $w$ satisfies the equation

$$
\Delta_{p} w(x, \tau)=e^{-\tau p \beta}\left(w_{\tau}-\alpha w\right) .
$$

We also remark that for $\tau \geq \tau_{0}$ sufficiently large, the solution $w(x, \tau)$ is positive inside $\Omega_{\tau}$, hence it is in $C^{1, \alpha}\left(\Omega_{\tau}\right)$ and all the calculations above are justified in the weak sense, together with the application of Proposition 6.1 We consider the function

$$
\Phi(x, \tau)=\frac{1}{T} \int_{\tau}^{\tau+T} w(x, s) d s-\frac{C_{\lambda_{0}} H_{p}(x)}{e^{\tau \beta(p-N) /(p-1)}}
$$

and we want to apply Proposition 6.1 to the function $\Phi$ in $\Omega_{\tau}$ (regarding $\tau$ as a frozen coefficient for the moment). Then $\Phi$ is a weak solution of the elliptic problem

$$
\left\{\begin{array}{l}
\Delta_{p} \Phi=\frac{1}{T} \int_{\tau}^{\tau+T} e^{-s p \beta}\left(w_{s}-\alpha w\right) d s=f(x, \tau) \quad \text { in } \Omega_{\tau}, \\
\Phi=0 \quad \text { on } \partial \Omega \cap \partial \Omega_{\tau}, \quad \Phi=h \quad \text { on } \partial B(0, R(\tau)) \cap \partial \Omega,
\end{array}\right.
$$


where

$$
h(x, \tau)=\frac{1}{T} \int_{\tau}^{\tau+T} g(x, s) d s-\frac{C_{\lambda_{0}} H_{p}(x)}{e^{\tau \beta(p-N) /(p-1)}} .
$$

By integrating with respect to $\tau$ in 6.10 , we derive easily that

$$
|h(x, \tau)| \leq\left|F_{\lambda_{0}}(\delta)-\frac{C_{\lambda_{0}} H_{p}(x)}{e^{\tau \beta(p-N) /(p-1)}}\right|+\varepsilon
$$

for all $x \in \Omega$ with $|x|=R(\tau)$. But

$$
\begin{aligned}
\mid F_{\lambda_{0}}(\delta)-\frac{C_{\lambda_{0}} H_{p}(x)}{e^{\tau \beta(p-N) /(p-1)} \mid \leq} & \left|F_{\lambda_{0}}(\delta)-C_{\lambda_{0}} \delta^{(p-N) /(p-1)}\right| \\
& +\left|C_{\lambda_{0}} \delta^{(p-N) /(p-1)}-\frac{C_{\lambda_{0}} H_{p}(x)}{e^{\tau \beta(p-N) /(p-1)}}\right|
\end{aligned}
$$

and obviously there exists $\delta>0$ sufficiently small and $\tau_{0}=\tau_{0}(\varepsilon, \delta)>0$ sufficiently large such that both terms on the right-hand side of the above inequality are less than $\varepsilon$ for $\tau \geq \tau_{0}$. We derive that $|h(x, \tau)| \leq 3 \varepsilon$ for all $\tau \geq \tau_{0}$ and $x \in \partial B(0, R(\tau)) \cap \partial \Omega$. In order to estimate $\left|\Delta_{p}(\Phi)\right|$, we perform an integration by parts, as in [14], and obtain

$$
\begin{aligned}
f(x, \tau)= & \frac{1}{T} e^{-\tau p \beta}\left(e^{-T p \beta} w(x, T+\tau)-w(x, \tau)\right) \\
& +\frac{1}{T}\left(p-k_{2}\right) \beta \int_{\tau}^{\tau+T} e^{-s p \beta} w d s,
\end{aligned}
$$

where $k_{2}=\alpha / \beta$ is the eigenvalue of the $p$-Laplace evolution equation corresponding to the dipole solutions (cf. [13]). Since $w$ is uniformly bounded in $\partial \Omega_{\tau}$ by a constant independent of $\tau$, it is easy to see that $w$ has the same property in the whole $\Omega_{\tau}$. Hence $|f(x, \tau)| \leq C e^{-\tau p \beta}$ for all $\tau \geq \tau_{0}$ sufficiently large. Using Proposition 6.1, we have

$$
|\Phi(x, \tau)| \leq C \delta^{p /(p-1)}+3 \varepsilon
$$

for all $x \in \Omega_{\tau}$ and $\tau \geq \tau_{0}>0$, with a constant $C$ independent of $\tau$. By choosing $\delta$ sufficiently small, we obtain the estimate 6.9 .

Passing from the convergence of the time averages to the convergence of $w$ is a standard thing (see [4]), hence we will only sketch it for the reader's convenience. We have:

Theorem 6.1. For any $\varepsilon>0$, there exists $\delta=\delta(\varepsilon)>0$ and a sufficiently large time $t_{\text {in }}=t_{\text {in }}(\varepsilon, \delta)$ such that

$$
\left|t^{\alpha} u(x, t)-\frac{C_{\lambda_{0}} H_{p}(x)}{t^{\beta(p-N) /(p-1)}}\right| \leq \varepsilon
$$

for all $t \geq t_{\text {in }}$ and $x \in \Omega$ with $|x| \leq \delta t^{\beta}$. 
Proof. We argue by contradiction and suppose that there exists a sequence $\left\{x_{n}, \tau_{n}\right\}$ such that

$$
\tau_{n} \rightarrow \infty, \quad \frac{\left|x_{n}\right|}{\delta e^{\tau_{n} \beta}} \rightarrow 0, \quad w\left(x_{n}, \tau_{n}\right) \geq \frac{C_{\lambda_{0}} H_{p}\left(x_{n}\right)}{e^{\tau_{n} \beta(p-N) /(p-1)}}+\varepsilon
$$

as $n \rightarrow \infty$. We use the Bénilan-Crandall estimate for solutions of the $p$-Laplacian equation (see [2] or [9]), and obtain $w_{\tau} \geq-C w$ for some constant $C>0$. By integrating on $\left(\tau_{n}, \tau_{n}+h\right)$, we obtain

$$
w\left(x_{n}, \tau_{n}+h\right) \geq\left(\frac{C_{\lambda_{0}} H_{p}\left(x_{n}\right)}{e^{\tau_{n} \beta(p-N) /(p-1)}}+\varepsilon\right) e^{-C h} .
$$

By integrating again over $h$ and performing straightforward calculations, we obtain in the end

$$
W_{T}\left(x_{n}, \tau_{n}\right) \geq \frac{C_{\lambda_{0}} H_{p}\left(x_{n}\right)}{e^{\tau_{n} \beta(p-N) /(p-1)}}+\frac{\varepsilon}{2}
$$

for $T$ sufficiently small, contradicting Proposition 6.2 .

\section{Global formulation}

In this section we gather the results of Theorems 6.1 and 3.1 into a global approximation result. In order to state it, we have to modify $H_{p}$ in the outer region, by defining

$$
\Psi(x, t)=\left\{\begin{array}{ll}
H_{p}(x) & \text { if }|x| \leq \delta t^{\beta}, \\
H_{p}\left(\delta t^{\beta} x /|x|\right) & \text { if }|x| \geq \delta t^{\beta}
\end{array} .\right.
$$

In this way, in the outer region, the inner approximant is made as small as we want. We are ready to state the global asymptotic behaviour theorem.

Theorem 7.1. Let $u$ be the unique solution of the problem 1.1 in dimension $N<p$ and

$$
V(x, t)=D_{\lambda_{0}}(x, t)+t^{-\alpha} \frac{C_{\lambda_{0}} \Psi(x, t)}{t^{\beta(p-N) /(p-1)}},
$$

where $\lambda_{0}$ and $C_{\lambda_{0}}$ are as in Section 6. Then

$$
\lim _{t \rightarrow \infty} t^{\alpha}|u(x, t)-V(x, t)|=0,
$$

uniformly for $x \in \Omega$. Moreover, we have

$$
\lim _{t \rightarrow \infty} t^{\left(k_{2}-N\right) \beta}\|u(x, t)-V(x, t)\|_{L^{1}(\Omega)}=0,
$$

where, as usual, $k_{2}=\alpha / \beta$. Both (7.3) and (7.4) can be extended to the whole class of solutions with initial data $u_{0} \in L^{1}(\Omega)$. 
Proof. (i) We work first with compactly supported initial data. Fix $\varepsilon>0$. We have

$$
t^{\alpha}|u(x, t)-V(x, t)| \leq\left|t^{\alpha} u(x, t)-\frac{C_{\lambda_{0}} \Psi(x, t)}{t^{\beta(p-N) /(p-1)}}\right|+t^{\alpha} D_{\lambda_{0}}(x, t) .
$$

But $t^{\alpha} D_{\lambda_{0}}(x, t)=F_{\lambda_{0}}\left(x t^{-\beta}\right)$ and, using the properties of the dipole profile, there exists $\delta=\delta(\varepsilon)>0$ sufficiently small such that $t^{\alpha} D_{\lambda_{0}}(x, t) \leq \varepsilon$ for $|x| \leq \delta t^{\beta}$ and $\delta \leq \delta(\varepsilon)$. From Theorem 6.1, there exists a time $t(\varepsilon)>0$ sufficiently large such that the first term on the right-hand side above is less than $\varepsilon$ for $t \geq t(\varepsilon)$ and $|x| \leq \delta t^{\beta}$. We obtain 7.3 in the inner region.

On the other hand, we have

$$
t^{\alpha}|u(x, t)-V(x, t)| \leq t^{\alpha}\left|u(x, t)-D_{\lambda_{0}}(x, t)\right|+\frac{C_{\lambda_{0}} \Psi(x, t)}{t^{\beta(p-N) /(p-1)}} .
$$

For $t$ sufficiently large and $|x| \geq \delta t^{\beta}$, the second term on the right-hand side above is less than $2 C_{\lambda_{0}} \delta^{(p-N) /(p-1)}$; indeed, this follows from the behaviour of the stationary solution $H_{p}$ near infinity. Hence, there exists $\delta(\varepsilon)>0$ such that this term becomes less than $\varepsilon$ for $\delta \leq \delta(\varepsilon)$. Using Theorem 3.1 for the first term on the right-hand side, we obtain 7.3 in the outer region $|x| \geq \delta t^{\beta}$.

We also remark that there exists $C>0$ such that $u(x, t)=V(x, t)=0$ for $|x| \geq C t^{\beta}$. Then, for $t \geq t(\varepsilon)$, we have

$$
\begin{aligned}
\int_{\Omega} t^{\left(k_{2}-N\right) \beta}|u(x, t)-V(x, t)| d x & =\int_{|x| \leq C t^{\beta}} t^{\left(k_{2}-N\right) \beta}|u(x, t)-V(x, t)| d x \\
& \leq C t^{\left(k_{2}-N\right) \beta} t^{-\alpha} \varepsilon t^{N \beta}=C \varepsilon,
\end{aligned}
$$

giving the convergence in the $L^{1}$ norm. We remark that 7.4 ) extends easily, by standard density arguments (see [19]), to the class of solutions with initial data $u_{0} \in L^{1}(\Omega)$.

(ii) We now prove 7.3 for solutions with initial data $u_{0} \in L^{1}(\Omega)$. The idea, which appeared already in [20] and [14], is to approximate $u_{0}$ from below by a compactly supported initial data $\tilde{u}_{0}$, such that the corresponding solutions satisfy

$$
\lim _{t \rightarrow \infty} t^{\left(k_{2}-N\right) \beta}\|u(t)-\tilde{u}(t)\|_{L^{1}(\Omega)}=0 .
$$

The existence of such an approximation follows from (7.4) and its extension to initial data in $L^{1}(\Omega)$, already proved. We pass to the renormalized variables and to the scaled versions $\tilde{u}_{\gamma}$ and $u_{\gamma}$. Fix $r>0$ larger than the radius of the support of $F_{\lambda_{0}}(\eta)$. In the ball of radius $r$ and time $1 / 2<t<1$ we have uniform convergence of $\tilde{u}_{\gamma}$ to $V(x, t)$ as $\gamma \rightarrow \infty$; hence, by the compactness of the orbit $u_{\gamma}$, we obtain the same result for the rescaled version of $u$. By setting $\gamma=t$, we obtain $\left[7.3\right.$, uniformly in $x \in \Omega,|x| \leq r t^{\beta}$. It remains to study the tail of $u$ for $|x| \geq r(t):=r t^{\beta}$. We do this directly; denote by $\Omega(t):=\left\{x \in \Omega:|x| \geq r t^{\beta}\right\}$ and fix $\varepsilon>0$. Then there exists a sufficiently large time $t(\varepsilon)>0$ such that $t^{\left(k_{2}-\bar{N}\right) \beta}\|u(t)\|_{L^{1}(\Omega(t))} \leq \varepsilon$ for any $t>t(\varepsilon)$. In order to transform this 
into a uniform convergence, we apply the $L^{1}-L^{\infty}$ smoothing effect (see [21, Chapter 11, Theorem 11.3]) to obtain

$$
\|u(t)\|_{L^{\infty}(\Omega(t))} \leq C\|u(t)\|_{L^{1}(\Omega(t))}^{\sigma} t^{-\alpha_{1}},
$$

where $\sigma=p \alpha_{1} / N$ and $\alpha_{1}$ is the exponent of the Barenblatt solution (see (1.6)). Taking into account the weighted $L^{1}$-convergence, we deduce that

$$
t^{\alpha}\|u(t)\|_{L^{\infty}(\Omega(t))} \leq C t^{\alpha-\alpha_{1}}\|u(t)\|_{L^{1}(\Omega(t))}^{\sigma} \leq C t^{\alpha-\alpha_{1}-\sigma \alpha+N \beta \sigma} \varepsilon^{\sigma}
$$

for any $t \geq t(\varepsilon)$. Using the general relation 1.10$)$ between the exponents, we obtain

$$
\begin{aligned}
\alpha-\alpha_{1}-\sigma \alpha+N \beta \sigma & =\alpha\left(1-\frac{p}{N(p-2)+p}\right)-\frac{N}{N(p-2)+p}+\frac{p N \beta}{N(p-2)+p} \\
& =\frac{N}{N(p-2)+p}((p-2) \alpha+p \beta-1)=0,
\end{aligned}
$$

hence

$$
t^{\alpha}\|u(t)\|_{L^{\infty}(\Omega(t))} \leq C \varepsilon^{\sigma} .
$$

Together with the previous considerations about the region $|x| \leq r t^{\beta}$, this ends the proof of (7.3) for solutions with $u_{0} \in L^{1}(\Omega)$.

Remark. The relation (7.4) illustrates the convergence in mass of $u$ to the dipole solution. Indeed, if we define $M(t)$ to be the mass of the dipole solution at time $t$, we find

$$
M(t)=\int_{\Omega} t^{-\alpha} F_{\lambda_{0}}\left(x t^{-\beta}\right) d x=t^{-\left(k_{2}-N\right) \beta} \int_{t^{-\beta} \Omega} F_{\lambda_{0}}(\eta) d \eta .
$$

Hence the rate of time $t^{\left(k_{2}-N\right) \beta}$ appearing in 7.4 is the exact decay power of the mass. Moreover, this improved convergence of the mass allows the application of the smoothing effect described in detail above.

Overlapping region. It is a region in $\Omega$ where both the inner and outer behaviours hold at the same time. This happens whenever the two asymptotic profiles $t^{\alpha} D_{\lambda_{0}}(x, t)$ and $C_{\lambda_{0}} H_{p}(x) / t^{\beta(p-N) /(p-1)}$ are very close. We work in the inner region $|x| \leq \delta t^{\beta}$. Then

$$
t^{\alpha} D_{\lambda_{0}}(x, t)=F_{\lambda_{0}}\left(|x| t^{-\beta}\right) \sim C_{\lambda_{0}} \delta^{(p-N) /(p-1)}
$$

for $\delta$ sufficiently small, fixed, and $t \geq t(\delta)$ sufficiently large. On the other hand,

$$
\frac{C_{\lambda_{0}} H_{p}(x)}{t^{\beta(p-N) /(p-1)}}=\frac{C_{\lambda_{0}} H_{p}(x)}{|x|^{(p-N) /(p-1)}}\left(|x| t^{-\beta}\right)^{(p-N) /(p-1)} .
$$

Fix $\varepsilon>0$; then there exists $C=C(\varepsilon)>0$ such that, using the properties of $H_{p}$ stated at the beginning of Section 4 , we have

$$
\frac{C_{\lambda_{0}} H_{p}(x)}{t^{\beta(p-N) /(p-1)}} \leq(1+\varepsilon) \delta^{(p-N) /(p-1)}
$$

for all $x \in \Omega$ with $C(\varepsilon) \leq|x| \leq \delta t^{\beta}$. By choosing $\delta$ small, we obtain an overlapping region for $1 / \delta \leq|x| \leq \delta t^{\bar{\beta}}$, for $\delta>0$ sufficiently small and $t>0$ sufficiently large. We illustrate the overlapping region in Figure 4 between the two dashed lines. 


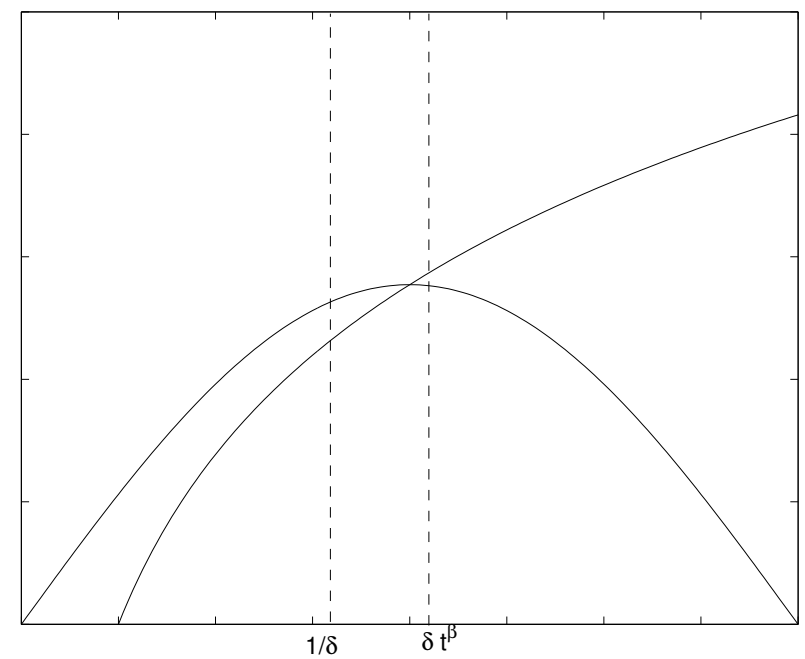

Fig. 4. Overlapping region.

\section{Application to the porous medium case}

(i) In this part we compare our results to the similar ones for the porous medium equation, where the critical dimension is $n=2$ (note that it is equivalent to take $p=2$ ). Hence the unique subcritical dimension which makes sense physically is $n=1$ (we make the convention that the dimension for the porous medium equation will be denoted by $n$ ), although in the radially symmetric setting any positive dimension is allowed. The outer analysis for dimensions $n \in(1,2)$ was performed by Gilding and Goncerzewicz in [11], using a very different technique, based on comparison principles associated to some weighted integrals of the solutions (that we do not have in our problem). The asymptotic behaviour is given by a dipole solution of the porous medium equation, having the general form

$$
\begin{aligned}
Z_{C}(x, t) & =t^{-\alpha} U\left(x t^{-\beta}\right), \\
U(\eta) & = \pm|\eta|^{(2-n) / m}\left(C-\frac{m-1}{2(n(m-1)+2)}|\eta|^{n+(2-n) / m}\right)_{+}^{1 /(m-1)},
\end{aligned}
$$

where $\alpha=1 / m$ and $\beta=1 / 2 m$ do not depend on the dimension $1 \leq n<2$. The form of the profile depends on $n$, but it is explicit in all cases. So there is no anomalous phenomenon.

(ii) Our techniques allow for a study of the inner behaviour, not covered in [11]. Since the adaptation presents no essential difficulties, we only state the final result. First, consider the solutions of the corresponding stationary problem

$$
\begin{cases}\left(H^{m}\right)_{x x}=0 & \text { in } \Omega, \\ H=0 & \text { on } \partial \Omega,\end{cases}
$$


which are $H_{C}(x)=C(x-1)^{1 / m}$ for any constant $C>0$, and fix $H(x)=H_{1}(x)$. The following result settles the inner behaviour of the solutions of the porous medium equation in dimension 1.

Theorem. Let $u$ be a solution of the one-dimensional porous medium equation

$$
u_{t}=\left(u^{m-1} u_{x}\right)_{x}
$$

in $Q=(1, \infty) \times(0, \infty)$, with initial data $u_{0} \in L^{1}((1, \infty))$ and non-negative. Then for any $\varepsilon>0$, there exists $\delta=\delta(\varepsilon)>0$ sufficiently small and a large time $t_{\text {in }}>0$ such that

$$
\left|t u^{m}(x, t)-\frac{C_{0}^{m}(x-1)}{t^{1 / 2}}\right| \leq \varepsilon
$$

for all $t \geq t_{\text {in }}$ and $x \in\left(1, \delta t^{\beta}\right)$.

Here, $C_{0}$ is the constant given by the matching with the outer behaviour given by the dipole solution (see [11]).

(iii) A different result concerning the porous medium equation can be obtained from our study using the correspondence relations between the $p$-Laplacian equation and the porous medium equation in [13]. Recall also that [3] studies a family of self-similar solutions of the porous medium equation, denoted by $U_{3, \lambda}$, which have lap number 2 , and it is proved that they are anomalous. Here $\lambda$ comes from a scaling similar to (1.12), with the precise formula

$$
U_{3, \lambda}(x, t)=\lambda^{2} U_{3}\left(\lambda^{1-m} x, t\right)
$$

We deal here with the exterior Neumann problem on a half line. More precisely:

Proposition. Consider, in dimension $n=1$, the solution $v$ of the exterior Neumann problem

$$
\left\{\begin{array}{l}
v_{t}=\left(|v|^{m-1} v\right)_{x x} \quad \text { in } \Omega \times(0, \infty), \\
v(x, 0)=v_{0}(x), \quad \forall x \in \Omega, \\
v_{x}(0, t)=0, \quad \forall t>0,
\end{array}\right.
$$

where $\Omega=(0, \infty)$ and $v_{0}$ is a continuous function with only one change of sign on the positive $x$ axis and with zero total mass, i.e. $\int_{\mathbb{R}} v_{0}(x) d x=0$. Then there exists $\lambda>0$ such that

$$
t^{\alpha_{3}}\left(v(x, t)-U_{3, \lambda}(x, t)\right) \rightarrow 0 \quad \text { as } t \rightarrow \infty
$$

with convergence in $L^{1}(\mathbb{R})$.

The proof is an immediate consequence of the results of the present paper and the fact that the solutions of the porous medium equation in dimension $n=1$ may be obtained from those of the $p$-Laplacian equation in dimension $N=1$ by differentiation (see [3] or [13] for more general correspondence relations).

Acknowledgments. Both authors were supported by Spanish Project MTM-2005-08760-C02. JLV was also partially supported by ESF Programme "Global and geometric aspects of nonlinear partial differential equations". The first author wishes to thank David Torres for his great help with the figures. 


\section{References}

[1] Barenblatt, G. I.: Scaling, Self-Similarity, and Intermediate Asymptotics. Cambridge Univ. Press, Cambridge (1996) Zbl 0907.76002 MR 1426127

[2] Bénilan, P., Crandall, M.: Regularizing effects of homogeneous differential equations. In: Contributions to Analysis and Geometry (Baltimore, MD, 1980), Johns Hopkins Univ. Press, Baltimore, 23-39 (1981) Zbl 0556.35067 MR 0648452

[3] Bernis, F., Hulshof, J., Vázquez, J. L.: A very singular solution for the dual porous medium equation and the asymptotic behaviour of general solutions. J. Reine Angew. Math. 435, 1-31 (1993) Zbl 0756.35038 MR 1203909

[4] Brandle, C., Quirós, F., Vázquez, J. L.: Asymptotic behaviour of the porous media equation in domains with holes. Interfaces Free Bound. 9, 211-233 (2007) Zbl 1131.35043 MR 2314059

[5] Chiarenza, F., Serapioni, R.: A Harnack inequality for degenerate parabolic equations. Comm. Partial Differential Equations 9, 719-749 (1984) Zbl 0546.35035 MR 0748366

[6] Chiarenza, F., Serapioni, R.: Pointwise estimates for degenerate parabolic equations. Appl. Anal. 23, 287-299 (1987) Zbl 0591.35031 MR 0875716

[7] Coifman, R. R., Fefferman, C.: Weighted norm inequalities for maximal functions and singular integrals. Studia Math. 51, 241-250 (1974) Zbl 0291.44007 MR 0358205

[8] DiBenedetto, E.: Degenerate Parabolic Equations. Series Universitext, Springer, New York (1993) Zbl 0812.35069 MR 1230384

[9] Esteban, J. R., Vázquez, J. L.: Homogeneous diffusion in $\mathbb{R}$ with power-like nonlinear diffusivity. Arch. Ration. Mech. Anal. 103, 39-80 (1988) Zbl 0683.76073 MR 0946969

[10] Friedman, A., Kamin, S.: The asymptotic behaviour of gas in an $N$-dimensional porous medium. Trans. Amer. Math. Soc. 262, 551-563 (1980) Zbl 0447.76076 MR 0586735

[11] Gilding, B., Goncerzewicz, J.: Large-time behaviour of solutions of the exterior-domain Cauchy-Dirichlet problem for the porous media equation with homogeneous boundary data. Monatsh. Math. 150, 11-39 (2007) Zbl pre05133243 MR 2297251

[12] Hulshof, J.: Similarity solutions for the porous medium equation with sign changes. J. Math. Anal. Appl. 157, 75-111 (1991) Zbl 0777.35034 MR 1109445

[13] Iagar, R., Sánchez, A., Vázquez, J. L.: Radial equivalence for the two basic nonlinear degenerate diffusion equations. J. Math. Pures Appl. 89, 1-24 (2008) Zbl 1139.35067| MR 2378087

[14] Iagar, R., Vázquez, J. L.: Asymptotic analysis of the $p$-Laplacian flow in an exterior domain. Ann. Inst. H. Poincaré Anal. Non Linéaire 26, 497-520 (2009) MR 2504041

[15] Kamin, S., Vázquez, J. L.: Fundamental solutions and asymptotic behaviour for the $p$ Laplacian equation. Rev. Mat. Iberoamer. 4, 339-354 (1988) Zbl 0699.35158 MR 1028745

[16] Kamin, S., Vázquez, J. L.: Asymptotic behaviour of solutions of the porous medium equations with changing sign. SIAM J. Math. Anal. 22, 34-45 (1991) Zbl $0755.35011 \mid$ MR 1080145

[17] Kamin, S., Peletier, L., Vázquez, J. L.: On the Barenblatt equation of elasto-plastic filtration. Indiana Univ. Math. J. 40, 1333-1362 (1991) Zbl 0737.73048 MR 1142718

[18] Vázquez, J. L.: A strong maximum principle for some quasilinear elliptic equations. Appl. Math. Optim. 12, 191-202 (1984) Zbl 0561.35003 MR 0768629

[19] Vázquez, J. L.: Asymptotic behaviour for the porous medium equation posed in the whole space. J. Evol. Equations 3, 67-118 (2003) Zbl 1036.35108 MR 1977429

[20] Vázquez, J. L., The Porous Medium Equation. Mathematical Theory. Oxford Math. Monogr., Oxford Univ. Press (2007) Zbl 1107.35003 MR 2286292

[21] Vázquez, J. L.: Smoothing and Decay Estimates for Nonlinear Diffusion Equations. Equations of Porous Medium Type. Oxford Univ. Press, Oxford (2006) Zbl 1113.35004 MR 2282669 